\title{
Applications of spatial models to ordinal data
}

$4 \quad{ }^{1}$ USDA, Agricultural Research Service, Plant Science Research Unit, 1991 Upper Buford Circle, Saint

5 Paul, MN, 55108 USA

$6{ }^{2}$ USDA, Agricultural Research Service, Corn Insects and Crop Genetics Research Unit, 819 Wallace

7 Road, Ames, IA, 50010 USA

$8{ }^{3}$ Department of Agronomy, Iowa State University, Ames, IA 50010 USA

$9 *$ Corresponding author wdbeavis@iastate.edu 


\section{Abstract}

11 Models have been developed to account for heterogeneous spatial variation in field trials. These

12 spatial models have been shown to successfully increase the quality of phenotypic data resulting

13 in improved effectiveness of selection by plant breeders. The models were developed for

14 continuous data types such as grain yield and plant height, but data for most traits, such as in iron

15 deficiency chlorosis (IDC), are recorded on ordinal scales. Is it reasonable to make spatial

16 adjustments to ordinal data by simply applying methods developed for continuous data? The

17 objective of the research described herein is to evaluate methods for spatial adjustment on ordinal

18 data, using soybean IDC as an example. Spatial adjustment models are classified into three

19 different groups: group I, moving average grid adjustment; group II, geospatial autoregressive

20 regression (SAR) models; and group III, tensor product penalized P-splines. Comparisons of eight

21 models sampled from these three classes demonstrate that spatial adjustments depend on severity

22 of field heterogeneity, the irregularity of the spatial patterns, and the model used. SAR models

23 generally produce better performance metrics than other classes of models. However, none of the

24 eight evaluated models fully removed spatial patterns indicating that there is a need to either adjust

25 existing models or develop novel models for spatial adjustments of ordinal data collected in fields

26 exhibiting discontinuous transitions between heterogeneous patches.

28 Abbreviations: iron deficiency chlorosis (IDC), geospatial autoregressive regression (SAR), 29 relative efficiency (RE), ordinary least square with range and row (OLS w/ RR), first-order 30 autoregressive (AR1) 


\section{Introduction}

Iron deficiency chlorosis (IDC) in soybeans is caused by the inability of the plant to utilize iron in

33 the soil. Without enough iron, chlorophyll production is hampered, and the plant will suffer and

34 possibly die. Symptoms of IDC are expressed in new leaf tissues of younger leaves, between the

35 first and third trifoliate growth stages, vegetative stages V1 to V3 [1]. The typical symptoms of

36 IDC is the yellowing of leaves, with interveinal chlorosis, while the veins remain green [2].

37 Soybeans are the second-most-planted field crop in the United States after corn, with a record-high

38 of 90.16 million acres planted in 2016 [3]. It is estimated that IDC reduces yields in farmers fields

39 by $20 \%$ each unit for each level of increased chlorosis scores. IDC is scored by field breeders on

40 a 1 (no yellowing symptoms) to 5 (severe yellowing of leaves, and the plant dies) scale [4].

41 Soybean planting acreage in IDC-prone regions has increased from 1979 to 2017, with a 160\%

42 increase of soybean production areas into IDC-prone regions in the past 30 years [5]. This increase

43 of soybean production area into IDC-prone regions has led to yield losses of 340 million tons,

44 worth an estimated $\$ 120$ million per year [6].

45 In the primary soybean production regions, soil micro-environmental variation results in location

46 heterogeneity for iron deficiency. Thus, it is difficult to find large and uniform fields of calcareous

47 soil that can be used to evaluate IDC in typical field plots, resulting in more experimental error

48 than is desirable for selecting resistant genotypes [7]. Environmental conditions for soybean to

49 develop IDC symptoms are ephemeral, usually existing for a couple weeks during the V1 to V3

50 stages of soybean development. Fields chosen for IDC testing are selected based on both historical

51 IDC pressure records and potential for IDC conditions detected at the time of planting. In fields

52 known to exhibit IDC, the exact locations within the fields may change from year to year, 
53 depending on rainfall prior to planting, and the rate at which soil moisture evaporates in the early

54 growing season. Within a testing site, IDC pressure usually varies either by ranges and rows from

55 year to year, leading to different levels and patterns of IDC expression with spatial autocorrelations.

56 To find the exact locations of IDC pressure within a field each year, IDC-susceptible varieties are

57 planted in plots throughout the field, augmenting the varieties planted in incomplete blocks. Seed

58 companies usually evalaute thousands of lines each year at each location, with at most two

59 replicates; thus some lines may be planted in high and some in low IDC-pressure areas. The

60 ephemeral nature of IDC spatial and temporal variation for high and low IDC pressure cannot be

61 planned for, given the small plot evaluations of early-stage field trials, and thus require spatial

62 models to correct for variation in IDC pressure within fields.

63 IDC phenotype scores typically range from 1 (the most resistant) to 5 (the most susceptible) in

64 reports by academic field breeders [8], or from 1 to 9 by some commercial plant breeding

65 organizations. In both cases, the scores represent ordinal data [9]. While plant breeders attempt to

66 create ordinal data that forces IDC values to behave as continuous variables, the actual IDC scores

67 often change in adjacent plots sharply from 1 to 9 . This is consistent with what is observed in

68 farmers fields. IDC symptoms in Iowa and southern Minnesota often appear to consist of oval

69 shaped patches (Fig 1), due to location of soil moisture. In summary, ordinal data such as IDC

70 creates phenotyping and selection challenges for plant breeders due to the genetic complexity of

71 the trait, the scoring of IDC as ordinal data, and the use of small plots with 3 to 8 plants in early-

72 stage evaluations in fields with ephemeral sharply delineated patches of IDC.

73 Fig 1. Spatial variation patterns in soybean IDC in commercial production fields. (A) IDC oval

74 pattern in the lower/ditch area at Madrid, Iowa in 2016. (B) IDC circle pattern in a farmer's field

75 at Ames, Iowa 2016. 
76 Accurate phenotypic data is the most important factor for both visual-based phenotype selection

77 and marker-assisted selection. High-quality phenotypic data relies on both experimental design

78 and accurate assessment of the phenotypes. Since presence of IDC is to some degree opportunistic,

79 standard IDC resistant and susceptible checks or controls are included in field trials to estimate

80 both the overall IDC pressure across the testing site and used as a reference to measure and adjust

81 IDC scores of previously untested genotypes. To balance the dilemma of arrangement of check

82 plots and maximize the number of test-line entries with field spatial variation, many experimental

83 designs, such as augmented design [10], modified augmented design [11, 12], partial replicated

84 [13], and augmented partially replicated (P-rep) designs [14-16] such as incomplete block alpha

85 lattice $[17,18]$, have been developed to account for field plot variation. Also, different statistical

86 models have been developed to account for field variation. Phenotype data quality control is the

87 process of removing non-genetic variation caused by environmental noise from the estimated

88 genotype values. Phenotypic data variation and subsequent patterns, or spatial variation, have been

89 studied for many decades, especially in the geostatistics and econometrics discisplines [19-23].

90 Spatial models are used to account for autocorrelation among neighbors, which violates the

91 identical independent distribution (iid) assumption for ordinary least square (OLS) analyses of

92 variance [24]. Various spatial adjustment techniques have been developed to account for the spatial

93 autocorrelation and significantly improve precision and repeatability of quantitative phenotypes.

94 Collectively, these models can be clustered into three groups of spatial models based on the time

95 of model development and the optimization mechanism used to adjust the spatial variation.

96 The first group includes the moving grid mean adjustment models [25-29]. The moving mean

97 spatial analysis recently has become popular because the R package "mvngGrAd" implements the

98 analysis. The package provides flexibility to pre-define any grid or pattern consisting of 
99 neighboring plots. The mean of the plots included in the grid is calculated and used as a covariate

100 to account for the spatial variation [30]. In contrast with the spatial autoregressive (SAR) model,

101 which treats spatial variation anisotropic along different directions [31], the moving mean average

102 models treat spatial variation as isotropic, in which the covariates among neighbor plots are simple

103 means of the neighbor plots within a user-defined grid. This approach has been reported to adjust

104 the spatial variation successfully and thus has increased genomic selection accuracy from 0.231 to

1050.37 for grain yield and from 0.436 to 0.614 for days to heading for wheat breeding projects [32].

106 The second and most extensively studied classification consists of the spatial autoregressive

107 regression (SAR) models. These include parameters for autocorrelations among neighboring plots

108 as covariates to model the correlated variation among neighbors. These models assme that closer

109 the neighbors will be more highly correlated [33]. Versions of these models include spatial lag

110 models, spatial error models, spatial lag plus error mixed models [34, 35], first-order

111 autoregressive regression $\mathrm{AR}(1)$ or one-dimensional spatial analysis with row, column, or row +

112 column [36], and the extended two-dimensional spatial analysis with interactions of rows and

113 columns [37].

114 The SAR models focus on optimizing the variance-covariance structure of the residuals among

115 neighbor plots. Evidence from a systematic comparison of covariance structures among

116 experimental, spherical, Gaussian, linear, linear log, anisotropic power, and anisotropic

117 exponential, show that AR(1) was generally not an optimal option for spatial analysis, and different

118 covariance structures are needed to account for spatial variation at different trial sites [38], because

119 each trial site has different variation patterns. Within this group of models, field variations are also

120 divided into local trend or small-scale variation within a block or experiment, and global trend or

121 large scale variation across the entire trial sites [39]. The nearest neighbor analysis model was 
122 developed to correct both local and global neighbors for the field variation [40, 41]. More complex

123 models with polynomials were also developed to account for additive effects for either row or

124 column, and non-additive epistasis interaction between the rows and columns [42]. To remove the

125 local and global field variation effectively, Gilmour et al. [43, 44]proposed a sequential spatial

126 model schema in which the first step removes local trend variation by fitting a two-dimensional

127 range by row $\mathrm{AR}(1)$ and the second step is to remove the global trend variation by fitting one-

128 dimensional polynomials or splines in the direction of rows or columns. To select the best model

129 for each trial site, the sequential spatial model will run both model selection and model variable

130 selection manually by applying graphical diagnostic tools to the spatial models. This manual model

131 selection process was further extended with more optional models for the comparison and

132 enhanced for model selection efficiency [45]. Over time the effectiveness of the spatial variation

133 correction of SAR models have been improved. SAR analyses are routinely used for data analyses

134 in geostatistics and econometrics [46].

135 The third group of models to account for spatial variation are known as "Tensor product penalized 136 spline models," or P-splines. These have been used to account for both local and global spatial

137 variation in tree genetics using mixed models in Bayesian methods. Application of these methods

138 to tree genetics increased the accuracy of estimated breeding values by $66 \%$ [47]. Bayesian mixed

139 model methods were extended to 2-dimensional smoothed surface in an individual-tree based

140 model using tensor product of linear, quadratic, and cubic splines for rows and columns, and the

141 accuracy of breeding values for the offspring increased by 46.03\% [48]. Most recently, an

142 advanced P-spline model was proposed and developed as the R package Spatial Analysis of field

143 Trials with Splines, "SpATS" [49]. This model includes both bilinear polynomial and smooth

144 splines components: 1) The bilinear polynomial component consists of three sub-variables: row 
145 spatial trend, spatial column trend, and interaction between row and columns; and 2) The smooth

146 spline component contains five smooth additive spatial components. This approach uses two-

147 dimensional P-spline ANOVA representation of the anisotropic smooth surface formulated in a

148 mixed model via SpATS. SpATS is advocated [49] because SpATS provides a one-step modeling

149 approach by fitting a general SpATS model to analyze all field trials. The SpATS approach

150 overcomes the sequential spatial model selection for different testing sites and can be used with

151 large-scale, high-throughput and routine analyses of multiple environmental trials (METs). SpATS

152 can fit both local and global variation, isotropic and anisotropic variation, one-dimensional and

153 two-dimensional variation with one model, and optimize the best estimates of parameters to

154 remove the noise from the true genotypic values without overfitting. With single step for model

155 fitting, it minimizes the chance of using different models selected for different trials - which might

156 lead to biases against different genotypes from different locations due to different selected model

157 variables [50]. SpATS was tested by modeling spatial trends in sorghum breeding fields, and the

158 results show that the improvement in precision and predicted genotypic values from SpATS

159 analysis were equivalent to those obtained using the best SAR sequential model selection for each

160 trial [50].

161 The three groups of spatial models for adjusting spatial variation have been mainly applied in

162 econometrics and geostatistics while a few have been applied by plant breederes for continuous

163 data, such as crop grain yield and plant height $[28,50,51]$. In contrast to continuous yield data,

164 soybean IDC scores are discrete ordinal variables, whereas moving grid, SAR and P-spline models

165 were developed and applied to continuous variables. In the applications, adjusted continuous trait 166 phenotypes have improved precision and the repeatability of the field trials. To our knowledge,

167 the application of these methods to adjust for non-genetic spatial patterns exhibited with ordinal 
168 traits such as IDC is limited to a single publication in which the moving grid was applied to IDC

169 scores in soybean [28]. Also, while most of the published reports about spatial analysis models

170 have investigated a few models, none have systematically compared the effectiveness of models

171 for data obtained from fields with different levels of severity and irregular discontinuous spatial

172 patterns. We hypothesize that the effectiveness of adjustments made by spatial models depends

173 not only on the model parameters but also the severity of the spatial variation and the irregularity

174 of variation patterns. The objective of research reported herein is: 1) to apply six different

175 geospatial as well as two OLS models to three datasets with different levels of severity of spatial

176 variation, variation patterns, field plot designs; and 2) to systematically compare models for spatial

177 adjustment using $\mathrm{R}^{2}$, AIC, residual standard error, Moran's I, and prediction accuracy. 


\section{Materials and methods}

180 A total of five data sets, four from field plot experiments, and one simulated provided a total of

181 11,602 unique genotypic lines (Table 1). Dataset 1 was simulated to mimic a circular IDC spatial

182 variation pattern that can be found in fields in which potholes were drained with tiles in the last 80

183 years. The parameters used for the simulation are summarized in Table 2. Dataset 1 contains 1,050

184 simulated genotypes with a total of 2,100 IDC scores. The field layout consists of 42 ranges by 50

185 rows and genotypes are assigned to plots using a randomized complete block design with two

186 replicates.

187 In contrast to simulated dataset 1, datasets 2 to 5 represesnted experimental field data from 2016.

188 They were selected to represent IDC-prone regions. For each set, the field plot design was a six189 by-six alpha-lattice consisting of 36 plots, that were assigned 32 testing lines and four checks.

190 These lines were in early stages of variety development projects, planted with two replicates in

191 one location. The average number of replicates was 1.84 replicates/line across all the experiments.

192 The reason for less than two replicates per testing line is due to low emergence rates at some sites.

193 IDC scores in data sets 2 to 5 were collected on individual hill plots from locations that have

194 historically provided expression of IDC in soybeans. These sites were selected to minimize the

195 IDC spatial variation by past years' IDC records and the current year's IDC status. Soybean IDC

196 pressures vary from year to year for the same site, and testing sites do not always provide

197 conditions for IDC expression every year. In order to assess potential IDC pressure at a site, several

198 varieties previously determined to be IDC susceptible were planted about 10 days earlier than the 199 expected planting date for all other lines. If the susceptible varieties showed IDC symptoms these 200 standard IDC controls were then rogued and new experimental lines were planted at testing sites.

201 Otherwise, these sites were not used as IDC testing sites. 
203 Table 1. Summary of the five data sets used to investigate application of geospatial models to 204 remove non-genetic spatial patterns of plots evaluated for iron deficiency chlorosis.

\begin{tabular}{|l|l|l|l|l|l|l|l|}
\hline $\begin{array}{l}\text { Dataset } \\
\text { Name }\end{array}$ & $\begin{array}{l}\text { Exp. } \\
\text { design }\end{array}$ & $\begin{array}{l}\text { No. } \\
\text { rows }\end{array}$ & $\begin{array}{l}\text { No. } \\
\text { range }\end{array}$ & $\begin{array}{l}\text { No. } \\
\text { entries }\end{array}$ & $\begin{array}{l}\text { Ave No } \\
\text { replicates }\end{array}$ & $\begin{array}{l}\text { No. } \\
\text { plots }\end{array}$ & $\begin{array}{l}\text { Data } \\
\text { sources }\end{array}$ \\
\hline Data set 1 & RCBD & 50 & 42 & 1,050 & 2.00 & 2,100 & simulated \\
\hline Data set 2 & $\alpha$-lattice & 220 & 26 & 2,774 & 1.83 & 5,074 & Iowa \\
\hline Data set 3 & $\alpha$-lattice & 24 & 220 & 2,652 & 1.79 & 4,740 & Minnesota \\
\hline Data set 4 & $\alpha$-lattice & 110 & 56 & 2,719 & 1.88 & 5,124 & Red River Valley \\
\hline Data set 5 & $\alpha$-lattice & 100 & 60 & 2,407 & 1.81 & 4,363 & Nebraska \\
\hline Total & & 504 & 404 & 11,602 & $\underline{\mathbf{1 . 8 4}}$ & 21,401 & \\
\hline
\end{tabular}

207 components, columns 2, 3, and 4 are the variance values, distribution used for the simulation, 208 and percentage of the variance components, respectively.

\begin{tabular}{|l|c|c|c|}
\hline Variance Component & Variance & Distribution & Percentage of SD (\%) \\
\hline Location SD & 1.5 & normal & 14.42 \\
\hline Experiment SD & 0.5 & Poisson & 4.81 \\
\hline Line SD & 2.1 & Normal & 20.19 \\
\hline Range SD & 0.2 & Normal & 1.92 \\
\hline Row SD & 1.0 & Normal & 9.62 \\
\hline Rep SD & 0.1 & Normal & 0.96 \\
\hline Pattern_SD & $\mathbf{3 . 8}$ & Normal & 36.54 \\
\hline Residual & 1.2 & Normal & 11.54 \\
\hline
\end{tabular}


210 IDC score scales from 1 for most tolerant to 9 for the most susceptible were used in all data sets.

211 The IDC score criteria are $1=$ green leaves (no chlorosis) to $9=$ dead plant, following the rating

212 scale presented in Fig 2. The IDC scores are treated as ordinal data.

213 Fig 2. IDC score reference from 1 to 9 as ordinal data. $1=$ green leaves (no chlorosis) to $9=$ dead plant.

214 Each image was taken from one hill plot with three to eight plants at growth stage V2 to V4.

215 Among the five data sets, we observed three classes of distinctive spatial variation patterns that we

216 refer to as the Red River Valley (RRV) pattern, the Iowa and Minnesota (IA/MN) pattern, and the

217 Kansas and Nebraska (KS/NE) pattern (Supplemental Fig 1). The three IDC field spatial patterns

218 are consistent with the three soybean IDC-prone soil types, which were clustered by principal

219 component analysis (PCA) based on 15 soil character measurements (Supplemental Fig 1).

220 Five testing sites from North Dakota and Manitoba were clustered as the RRV group, four testing

221 sites from Iowa and Minnesota were clustered together as the IA/MN group, and one testing site

222 from Nebraska represented the KS/NE group (Supplemental Fig 1). KS/NE regions have relatively

223 uniform IDC scores without noticeable spatial patterns, and no spatial model is needed to adjust

224 plot IDC scores in this region.

226 The Red River Valey (RRV) IDC data has spatial variation among columns represented as block

227 effects. In contrast, the IA/MN IDC testing sites show distinct spatial patterns relative to RRV and

$228 \mathrm{KS} / \mathrm{NE}$ regions. Thus, IDC data from the IA/MN region has spatial autocorrelation and needs

229 spatial autoregressive analysis. Among the five testing sites from the IA/MN region, two show

230 different spatial variation patterns (data sets 2 and 3) and are used to evaluate the geo spatial

231 methods. All the results reported hereafter use these three data sets: two data sets from the IA/MN

232 region, and one simulated data set (Table 1). 
234 Including ordinary linear square (OLS), a total of eight models were compared (Table 3).

235 Table 3. Summary of the eight models compared in the research.

\begin{tabular}{|l|l|l|l|l|}
\hline $\begin{array}{l}\text { Model } \\
\text { Name }\end{array}$ & $\begin{array}{l}\text { Model } \\
\text { No }\end{array}$ & $\begin{array}{l}\text { Spatial } \\
\text { Term }\end{array}$ & $\begin{array}{l}\text { R } \\
\text { package }\end{array}$ & Reference \\
\hline OLS w/o RR & M1 & None & RMS & {$[52]$} \\
\hline OLS w/ RR & M2 & None & RMS & {$[52]$} \\
\hline MovingGrid & M3 & Mean of grid & mvngGrAd & {$[53]$} \\
\hline SAR + lag & M4 & Lag & spdep & {$[54]$} \\
\hline SAR + error & M5 & Error & spdep & {$[54]$} \\
\hline SAR Durbin & M6 & Lag+ Error & spatialreg & {$[55]$} \\
\hline ASReml AR1 & M7 & AR1(range): AR1(row) & ASReml-R & {$[56]$} \\
\hline P-spline & M8 & psanova(range, row) & SpATS & {$[57]$} \\
\hline
\end{tabular}

237 Model 1: Ordinary least square (OLS) without range and row covariates. OLS is a linear least-

238 square method for estimating the unknown parameters in a linear regression model. OLS chooses

239 the parameters of a linear function representing a set of explanatory variables by the principle of

240 least squares. Traditionally the regression model does not include parameters for the spatial

241 dependence of the experimental units. The general equation for the OLS with $p$ variables can be

242 written as:

$243 \quad \mathrm{Y}=\alpha \mathrm{L}_{\mathrm{n}}+\mathrm{X} \beta+\varepsilon \quad[46]$

244 Where $\mathrm{Y}$ and $\varepsilon$ are $(\mathrm{n} \times \mathrm{1})$ vector of the values of the response variable and the errors for the

245 various observations, respectively. $L_{n}$ is a $(n \times 1)$ vector of ones associated with the constant term 
246 parameter $\alpha$ to be estimated. $X$ is an $(n \times p)$ matrix of regressors or the design matrix. $\beta$ is $(1 \mathrm{p} \times 1)$

247 vector of the parameters to be estimated.

248 OLS analysis for model 1 was conducted with the ols() function implemented the R package

249 “regression modeling strategies (rms)" [52].

250 Model 2: Ordinary least square (OLS) with range and row. Model 2 analysis was similar to model 1

251 except two variables, range, and row were added in the model [45].

$252 \mathrm{Y}=\alpha \mathrm{L}_{\mathrm{n}}+\mathrm{X} \beta+\mathrm{ROW}+\mathrm{RANGE}+\varepsilon[46]$

253 Model 3: Moving grid adjustment. The "moving grid average adjustment" is a spatial method to adjust

254 for environmental variation in field trials. It is most common in field trials with few replicates, such as for 255 early-stage breeding materials. All the raw data were aligned into a row by range rectangle layout. A grid 256 is predefined based on the field variation pattern around each cell (= entry), and each observed value was 257 adjusted by the values from the neighbor plots within the predefined grid. The mean of the cells included 258 in the grid is calculated using the equation below [58]:

$$
x_{i}=\frac{\sum_{j} p_{j, o b s} \cdot I\left(p_{j, o b s} \in G_{i}\right)}{\sum_{j} I\left(p_{j, o b s} \in G_{i}\right)}, P_{i, o b s}=P_{i, o b s}-\mathrm{b}\left(x_{i}-\bar{x}\right)
$$

260 Where

$261 x_{i}$ is the moving mean of the ith entry

$262 G_{i}$ is the grid of entry $\mathrm{i}$ and $\mathrm{I}(\cdot)$ is an indicator function that takes the value " 1 " if the condition is satisfied

263 and " 0 " if not

$264 P_{j, o b s}$ are the observed phenotypic values of all entries which are included in $G_{i}$

$265 \bar{x}$ is the mean of all $x_{i}$

$266 \mathrm{~b}$ is the regression coefficient in the linear model.

$267 P_{i, o b s}$ are the adjusted phenotypic values of all entries. 
The layout of the grid used to adjust the phenotype value is shown below in Fig 3:

270 Fig 3. Layout of the grid used to calculate the mean.

272 The model executed for each data set was:

273 movingGrid (rows $=$ no.of.rows,

columns $=$ no.of.range,

$$
\text { layers }=c(1: 1)
$$

where "shapeCross" is to set up the shape of the moving grid, "excludeCenter" is to define whether the center from each grid is included/excluded to calculate the mean.

Model 4: Spatial autoregressive lag model. When a value in one plot depends on the values of

287 components were specified on the dependent variable, IDC scores. This setting leads to a spatial

288 filtering of the variable, which are averaged over the surrounding neighborhood defined in W,

289 called the spatially lagged variable. The spatial lag model can be specified as: 
291 Where $\rho$ is the autoregressive lag coefficient, which tells us how strong the resemblance is, on

292 average, between $Y_{i}$ and its neighbors; if $\rho$ is not significantly different from 0 , then spatial lag

293 model becomes traditional OLS regression model.

$294 y_{i}$ is the $i^{\text {th }}$ IDC score, and $y_{j}$ is all the neighbor's IDC scores around $\mathrm{i}^{\text {th }}$ IDC score. $y_{i}$ stands for

295 one of n observed IDC scores, $y_{j}$ stands for more than one IDC scores.

$296 \mathrm{~W}_{\mathrm{Y}}$ is a spatial weight matrix with $\mathrm{n} \times \mathrm{p}$ rows and ranges, describing the spatial correlation

297 structure of the observations.

$298 \mathrm{X}$ is an $\mathrm{n} \times \mathrm{p}$ matrix of regressors or the design matrix; $\beta$ is $\mathrm{p} \times 1$ vector of estimated coefficients.

299 Analysis of IDC scores with the spatial lag model was conducted via R package "spdep" [54] and

300 "spatilreg" [55].

301 Model 5: Spatial autoregressive error model. In contrast to the spatial lag model treating

302 autocorrelation as a lag component in the response variable, spatial error model regards the

303 autocorrelation as part of the error term. The spatial error model incorporates a local and a

304 spillover element in the variance-covariance matrix of the error term in a linear regression model

305 [61]. Formally, the model can be written as:

$306 \quad \mathrm{Y}=\mathrm{X} \beta+e$ and $\mathrm{e}=\lambda \mathrm{W}_{\mathrm{e}}+\mathrm{V}$

307 Where $\lambda$ is the spatial error coefficient, if the absolute value of the $\lambda$ is not significant bigger than

3080 , the spatial error model becomes the OLS regression model. $W_{\mathrm{e}}$ are the weight matrix to adjust

309 the error correlation in the residuals. 
310 Analysis of IDC scores with the spatial error model was conducted via R package "spdep" [54] and

311 "spatilreg” [55].

312 Model 6: Spatial Durbin mixed model. A limitation of the spatial lag or spatial error models is

313 that they can include either an autoregressive lag or a spatial error covariate in the model. In

314 reality, some fields have complexed spatial variation with both autocorrelation lag and spatial

315 error. Also, the dependencies in the spatial autoregressive relationships don't only occur in the

316 dependent variable but may also be present in the independent variables. The spatial Durbin

317 mixed model was developed to account for dependent, the autocorrelation lag, spatial error, and

318 independent variables $[62,63]$. The model can be written as:

$319 \mathrm{Y}=\rho W \mathrm{Y}+X \beta+W X \theta+\varepsilon$

320 Where

$321 \mathrm{Y}, X, \rho$, and $\beta$ are defined as above

$322 W Y$ : is the spatially lagged offering IDC scores accounting for various spatial dependencies

323 with $W$ defined as (n x n) spatial weight matrix

$324 \rho W Y$ : Endogenous interaction effect

$325 \theta:(\mathrm{k} \times 1)$ vector of unknown parameters

$326 \theta W X$ : Exogenous interaction effect

327 Implementation of the spatial Durbin model was carried out via the R package "spatilreg" [55].

328 Model 7: ARl by AR1 via ASReml-R. The ASReml mixed model is widely used in plant and

329 animal breeding and quantitative genetics. It also provides functions for spatial autoregressive

330 analysis. ASReml-R is the only commercial R package in this study and used to compare

331 whether it will outperform the free spatial analysis packages. One update in the new ASReml-R 
332 version 4 for spatial analysis, ASReml changed the random formula and error (rcov) component

333 "rcov $=\operatorname{ar1}($ range $): \operatorname{ar1}($ row $) "$ to residual $=\operatorname{ar1}($ range $): \operatorname{ar} 1($ row $)[56,64]$.

334 The model used for the analysis is: asreml(fixed $=$ IDC_scores $\sim 1$, random $=\sim$ LINCD, residual $=$ $335 \sim \operatorname{ar1}($ range):ar1(row), data $=$ IDC.data)

336 Model 8: P-spline mixed model implemented wth the R package SpATS. The P-spline approach

337 models field trends using a smooth bivariate function of the range and row $\mathrm{f}($ range, row),

338 represented by a 2D P-splines $[49,65]$. The P-spline method optimizes the fitted surface by

339 penalizing the spatial effects. The degree of penalization over the fitted spatial variation trend is

340 determined by smoothing parameters. The $2 \mathrm{D}$ range by row surface is decomposed into a sum of

341 linear components and univariate and bivariate smooth function as:

$342 \mathrm{f}($ range, row $)=X_{s} \beta_{s}+Z_{s} \mathrm{~s}[65]$

343 where:

$344 X_{s} \beta_{s}=\beta_{s 1}$ row $+\beta_{s 2}$ range $+\beta_{s 3}$ row . range

$345 Z_{s} \mathrm{~s}=f_{1}($ row $)+f_{2}($ range $)+h_{3}($ row $)$ range $+h_{4}($ range $)$.row $+f_{5}($ row , range $)$

$346 \beta_{\text {s1 }}$ row: linear trend by row

$347 \quad \beta_{s 2}$ range: linear trend by range or column

$348 \beta_{s 3}$ row. range: linear interaction trend by row $\mathrm{x}$ range

$349 f_{1}($ row $)$ : main smooth trend across rows

$350 f_{2}$ (range): main smooth trends across ranges

$351 h_{3}$ (row).range: interaction trends between linear range by smooth surface row

$352 h_{4}($ range $)$.row: interaction trends between linear row by smooth surface range

$353 f_{5}$ (row, range): smooth-by smooth trends between ranges and rows 
355 The P-splines mixed model can be written as:

$356 \mathrm{Y}=\mathrm{X} \beta+X_{s} \beta_{s}+Z_{s} \mathrm{~s}+Z_{u} u+Z_{g} g+\mathrm{e}[65]$

357 Where:

$358 \mathrm{X}, \beta, X_{s} \beta_{s}, Z_{s}$ s are the same as above

$359 Z_{u} u$ : $\mathrm{u}$ is the sub-vector of random row and range effects accounting for discontinuous field

360 variation; $\mathrm{Zu}=\left[Z_{\text {row }} \mid Z_{\text {range }}\right]$ is the design matrix

$361 Z_{g} g: \mathrm{g}$ is the vector of random effects of genotypic effects of the testing lines or hybrids; $Z_{g}$ are

362 the design matrix for the genotype effects.

363 The final SpATS model R scripts used in this study for P-spline is as below:

365 SpATS(response="IDC_Scores", genotype="LINCD", genotype.as.random = TRUE,

$$
\text { degree }=3 \text {, nest.div }=2 \text { ), fixed }=\text { NULL, }
$$$$
\text { control }=\text { list }(\text { tolerance }=1 \mathrm{e}-03, \text { monitoring }=1) \text {, }
$$

371 The term "spatial" is an auxiliary function used for modeling the spatial or environmental effect

372 as a two-dimensional penalized tensor-product of marginal B-spline basis functions with

373 anisotropic penalties based on the PSANOVA approach [66, 67]. Inside spatial, "nseg" stands for

374 the number of segments in the P-splines, 10 and 10 segments were used for both range and row, 
375 respectively. Parameter "degree" stands for numerical order of the polynomial of the B-spline basis

376 for each marginal. Degree of 3, cubic B-splines was used for the IDC analysis. Parameter "nest.div"

377 is a divisor of the number of segments (nseg) to be used for the construction of the nested B-spline

378 basis for the smooth-by-smooth interaction component. In this case, the nested B-spline basis will

379 be constructed assuming a total of nseg/nest.div segments. The value was set to 2 for the IDC

380 analysis.

\section{$381 \quad$ Performance metrics}

382 The effectiveness of models were compared using: 1) coefficient of determination, $\mathrm{R}^{2}$; 2) Akaike's

383 information criterion (AIC); 3) residual standard error (RSE); 4) Moran's I index; 5) p-value of

384 Moran's I; 6) prediction accuracy I (whole data set); 7) and prediction accuracy II (cross-prediction

385 accuracy).

386 All except Moran's Index are broadly used metrics for many statistical methods. Moran's index

387 also known as the spatial autocorrelation index simultaneously measures spatial autocorrelation

388 based on both feature locations and feature values [69]. With a set of features and an associated

389 attribute, Moran's I index evaluates whether the pattern expressed is clustered, dispersed, or

390 random. Moran's I test provides a way to check whether there is spatial autocorrelation in the field

391 data and whether residuals from a spatial model are not correlated or randomly distributed, with

392 the iid property. Moran's I test value is between -1 to +1 . A value of "-1" indicates the large and

393 small values intersperse across the field and the data are negatively auto-correlated, while " +1 "

394 indicates high IDC scores surrounded by high IDC scores or low IDC scores surrounded by low

395 IDC score; these scores are positively auto-correlated. If all the residuals from a model are iid, and

396 there is no autocorrelation, then Moran's I should be close to zero. If I is close to 0, then the spatial 
397 adjustment is successful. If I is close to either -1 or +1 , with $p$-values $<0.05$, then interpretation is

398 that the adjustment by the model does not remove a significant amount of the spatial

399 autocorrelation

400 Software that implements these models do not generate estimates of all of these metrics, thus the

401 metrics were calculated manually:

402

1) $R^{2}: R^{2}=1-\mathrm{SS}_{\text {residual }} / \mathrm{SS}_{\text {total }}[68]$

403 where $S S_{\text {residual }}$ is the sum of squares of the residual from the model, and $S S_{\text {total }}$ is the total

404 sum of squares from the data.

405

2) $A I C: A I C=2 k-2 \ln (\mathcal{L})=-2($ log-likelihood $)+2 K \quad[68]$ intercept). Log-likelihood is a measure of model fit. The higher the number, the better the fit. For AIC, the smallerthe value, the better fit of the model.

3) Residual standard error $(\mathrm{RSE})=$ square.root $(\mathrm{MSE})$ where MSE is mean square error.

4) Moran's I index: Moran's I index was calculated as:

Moran's I index $=\frac{N}{\sum_{i} \sum_{j} w_{i, j}} \frac{\sum_{i} \sum_{j} w_{i, j}\left(X_{i}-\bar{X}\right)\left(X_{j}-\bar{X}\right)}{\sum_{i}\left(X_{i}-\bar{X}\right)^{2}}$ one in the weight matrix of the rectangle): 
417 Where $\mathrm{N}$ is the number of spatial units indexed by $\mathrm{i}$ and $\mathrm{j} ; \mathrm{X}$ is IDC score; $\bar{X}$ is the average 418 IDC scroe; $w_{i, j}$ is the spatial weight matrix..

419 5) P-value of Moran's I: The null hypothesis for the Moran's I test in that there is no 420 autocorrelation among the data in the area, and the data collected are randomly distributed. If 421 the p-value from Moran's I test is not significant, or the p-value $>0.05$, the spatial distribution 422 of feature values may be the result of random spatial processes.

423 6) Prediction accuracy I (whole data set): is calculated as

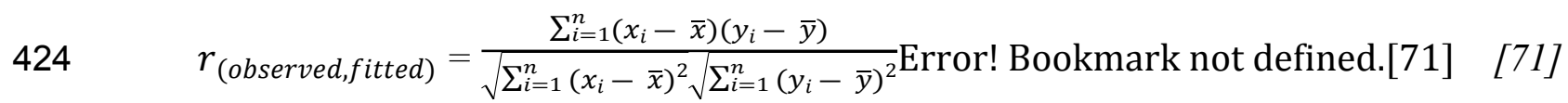

$425 \quad$ Where

$426 \mathrm{n}$ is the number data points or sample size

$427 \quad x_{i}$ from 1 to $\mathrm{n}$ is the observed values

$428 y_{i}$ from 1 to $\mathrm{n}$ is the fitted values from model

429 7) Prediction accuracy II (cross prediction): is the correlation coefficient between the overserved 430 and predicted for the testing lines planted in low IDC pressure area. Formula for prediction 431 accuracy II is the same as prediction accuracy I except using the testing lines planted in the $432 \quad$ low IDC pressure regions.

\section{Comparison methods}

434 Heatmap and Lagrange Multiplier Test. Heatmaps of IDC field layout were made using the 435 python package "seaborn" and the R package "fields" [72]. The Lagrange Multiplier Test was 436 conducted to select the best model among all the spatial autoregressive (SAR) models via function 437 "lm.LMtests “ from the R "spdep” package. 
439 the relative efficiency (RE) of each model relative to the OLS models:

$440 \quad$ Relative efficiency $=\frac{\text { MSE of the } O L S}{M S E \text { of another model }}[73]$

442 Results and discussion

\section{Assessments of data set complexity.}

444 Spatial effective dimension is a measure of the complexity, in which larger EDs are indicative of

445 more complex patterns [49]. The shapes of evident patchy spatial patterns of the IDC scores were

446 best explained by the integration of one-dimensional range or row trends (functional trend row or

447 range and surface range or row) and two-dimensional trends (interaction function trend row by

448 range "Row: Range," surface trend range by linear function trend row "F(Range): Row," linear

449 functional trend range by surface trend row "Range: F(Row)," and surface trend interaction range

450 by row "F(Range): $\mathrm{F}($ Row)" (Table 4). Overall, one-dimension surface range trends were more

451 complicated than that of row, in which all the $\mathrm{f}($ range $)>\mathrm{f}($ row $)$. For data set $1 \mathrm{f}($ range $) 3.0>$

$452 \mathrm{f}($ row $)=2.3$, for data set 2 the values are $7.0>6.6$, and for data set $35.8>3.3$ (Table 4). From the

453 two-dimension level, 2D surface trend rows by surface trend ranges " $\mathrm{f}($ row $): \mathrm{f}($ range $)$ " are the most

454 significant spatial terms. The percentage of the effective spatial dimensions are 58.42, 44.12, and

$45553.92 \%$ for data sets 1,2, and 3, respectively. Another observation is that linear trend for row plays

456 a large role (13.9 in data set 2) and linear trend range did not contribute at all (0 in data set 2).

457 Opposite results were obtained from data set 3: 24.6 for the linear trend of range and 17.6 for the

458 linear trend for row. The differences of linear trend ranges and rows between data sets 2 and 3

459 indicate the spatial structures of data set 2 and 3 are different. 
Table 4. Summary of effective spatial dimensions (EDs) of the smooth surface components for the

461 three data sets.

462

\begin{tabular}{|l|c|c|c|c|c|c|}
\hline Variables Name & \multicolumn{2}{|c|}{ Data Set 1 } & \multicolumn{2}{c|}{ Data Set 2 } & \multicolumn{2}{c|}{ Data Set 3 } \\
\hline & ED $_{\mathbf{s}}$ & $\mathbf{E D}_{\mathbf{m}}$ & $\mathbf{E D}_{\mathbf{s}}$ & $\mathbf{E D}_{\mathbf{m}}$ & ED $_{\mathbf{s}}$ & $\mathbf{E D}_{\mathbf{m}}$ \\
\hline Range & 10.1 & 42 & 0.0 & 220 & 24.6 & 221 \\
\hline Row & 41.1 & 50 & 13.9 & 28 & 17.6 & 24 \\
\hline Row:Range & 1.0 & 1 & 1.0 & 1 & 1.0 & 1 \\
\hline F(Range) & 3.0 & 11 & 7.0 & 11 & 5.8 & 11 \\
\hline F(Row) & 2.3 & 11 & 6.6 & 11 & 3.3 & 11 \\
\hline F(Range):Row & 0.0 & 11 & 6.5 & 11 & 5.8 & 11 \\
\hline Range:F(Row) & 1.8 & 11 & 1.6 & 11 & 10.0 & 11 \\
\hline F(Range):F(Row) & 83.3 & 121 & 28.9 & 36 & 79.7 & 121 \\
\hline \% F(Range):F(Row) & 58.42 & 46.90 & 44.12 & 10.94 & 53.92 & 29.44 \\
\hline Total & 142.6 & 258 & 65.5 & 329 & 147.8 & 411 \\
\hline
\end{tabular}

463 Results of variance components analysis of the three random (LINCD, Range, and Row), five 464 surface variables (f(RANGE), $\mathrm{f}(\mathrm{ROW}), \mathrm{f}(\mathrm{RANGE})$ : ROW, RANGE:f(ROW)), and one 2D surface 465 range trend by surface row trend, $f(R A N G E): f(R O W))$ are summarized in Table 5. These show 466 that: 1) three datasets have large differences in level of spatial variances, with dataset 1 having the 467 smallest total variance of 431.5 (Table 5) and dataset 2 having the largest variance of 6597.2 (15468 fold larger than that of dataset 1); 2) surface range trend by surface row trend $\mathrm{f}(\mathrm{RANGE}): \mathrm{f}(\mathrm{ROW})$, 469 accounts for the majority of the variance in dataset 1 and $2-$ up to $85.6 \%$ and $89.6 \%$, respectively; 470 and 3) dataset 3 has different spatial variation patterns from datasets 1 and 2; a smaller variance 471 component from surface range trend by surface row trend of $15.14 \%$. In contrast, linear range trend 472 by surface row trend, range:f(row), takes up to $78.1 \%$ in the dataset 3 . One observation is that 473 variance components for LINCDs are very small: $0.1 \%, 0.01 \%$, and $0.05 \%$ for datasets 1,2 , and 4743 , respectively. The overall mean-variance components for LINCDs is only $0.05 \%$ across the three 
475 data sets. Similar results were observed for the residual variance, in which the mean percentage of

476 variance component is only $0.1 \%$ across three datasets (last column in Table 5). The overall results

477 from the variance components analysis show that spatial variation along the rows are much larger

478 than that from ranges. The mean percentages related to surface row term " $\mathrm{f}(\mathrm{ROW})$ " are $5.95 \%$ for 479 “f(ROW)," 26.42\% for "RANGE:f(ROW)," and 63.44\% for "f(RANGE):f(ROW)" with a total of $480 \quad 95.81 \%$ (last column in Table 5).

481 Table 5. Summary of variance component analysis from the tensor product panelized P-splines.

482 Var stands for variance, type for data type: "R" for random and "S" for surface variable.

\begin{tabular}{|l|c|r|r|r|r|r|r|r|}
\hline \multirow{2}{*}{ Variables } & Type & \multicolumn{2}{|c|}{ Data set 1 } & \multicolumn{2}{|c|}{ Data set 2 } & \multicolumn{2}{|c|}{ Data set 3 } & Mean \\
\hline & & Var & \% var & Var & \% var & Var & \% var & \% var \\
\hline LINCD & R & 0.43 & 0.10 & 0.71 & 0.01 & 0.46 & 0.05 & 0.05 \\
\hline Range & $\mathrm{R}$ & 0.01 & 0.00 & 0.00 & 0.00 & 0.02 & 0.00 & 0.00 \\
\hline Row & $\mathrm{R}$ & 0.07 & 0.02 & 0.20 & 0.00 & 0.78 & 0.08 & 0.03 \\
\hline f(RANGE) & $\mathrm{S}$ & 4.97 & 1.15 & 39.05 & 0.59 & 13.09 & 1.39 & 1.04 \\
\hline f(ROW) & $\mathrm{S}$ & 55.63 & 12.89 & 223.32 & 3.39 & 14.92 & 1.58 & 5.95 \\
\hline f(RANGE):ROW & $\mathrm{S}$ & 0.31 & 0.07 & 352.16 & 5.34 & 32.58 & 3.45 & 2.95 \\
\hline RANGE:f(ROW) & $\mathrm{S}$ & 0.55 & 0.13 & 66.26 & 1.00 & 737.68 & 78.13 & 26.42 \\
\hline f(RANGE):f(ROW) & $\mathrm{S}$ & 369.15 & 85.56 & 5912.83 & 89.63 & 142.97 & 15.14 & 63.44 \\
\hline Residual & $\mathrm{R}$ & 0.34 & 0.08 & 2.61 & 0.04 & 1.67 & 0.18 & 0.10 \\
\hline total & & 431.47 & 100.00 & 6597.15 & 100.00 & 944.18 & 100.00 & 100.00 \\
\hline
\end{tabular}

485 The relative rankings for the models based on different evaluation metrics indicates each model 486 has advantages and disadvantages for correcting spatial patterns in the IDC data. A summary of 487 performance metrics for the eight models applied to data set 1 in Table 6 suggests that the P-spline 488 model implemented with SpATS provides the largest $\mathrm{R}^{2}$ of 0.8931 , prediction accuracy of 0.9473 , 
and residual standard error of 0.5582 . In contrast, the moving grid adjustment is the least desired

490 model because it has the smallest $\mathrm{R}^{2}(0.3409)$, lowest prediction accuracy (0.6246), and the largest

491 Moran's I index (0.9492). For data set 1, model OLS with range and row is the second-best based

492 on prediction accuracy (0.9200), which is much better than OLS without range and row in the

493 model indicating that row and range terms are important for correcting the spatial variation

494 associated with rows and ranges of the grid. Another observation is that ASReml has the second

495 largest $\mathrm{R}^{2}(0.7289)$ and second smallest residual standard error (0.6364), but prediction accuracy

496 (0.8538) is low and ranked the sixth among the eight models. If Moran's I index is used to rank

497 the models, SAR + mixed model is the best since it has the smallest Moran's I index (0.0349) and

498 the largest $\mathrm{p}$-value of the Moran's I index (0.02179). For all eight models, estimates of prediction

499 accuracy II is lower than that of prediction accuracy I indicating that the testing materials planted

500 in high IDC pressure areas have higher prediction accuracy than those planted in low or no IDC

501 pressure areas. AIC values are widely disparate among the models. For example, only two

502 parameters are used for the moving grid average, while the P-spline, requires many more

503 parameters: 142.6 for data set 1 and 147.8 for data set 3 (Table 4) to fine-tune the boundary of the

504 spatial trends and thus has a much larger AIC than the other models.

505 The results from Table 6 are generally consistent with the eight heatmaps of the residual plots from

506 data set 1 (Fig 4). The residual heatmap from model 1, 2, 4, 5, and 6 show a "baseball" pattern,

507 indicating little improvement in autocorrelations among the plots, whereas models 3, 7, and 8 show

508 a circle pattern, indicating better adjustments to remove autocorrelations among the residuals. The

509 heatmap from using the P-Spline model provides the greatest number of plots with random

510 residuals, and its legend bar has the smallest scales (from -2 to 1), whereas the rest of the models

511 have scales ranging from from -2 to 2 or 3 . The residual heatmap from "MovingGrid" is the typical 
512 case for positive autocorrelation close to 1 , where all the residuals inside the circle are -1 values

513 of +3 outside the circle. Moran's I index of the residual heatmap from "MovingGrid" model also

514 is the largest and is close to 1 . Based on estimated performance metrics in Table 6 and Fig. 4, it

515 appears that the best models for adjusting plot IDC scores in data set 1 are either the "SAR + mixed"

516 and "P-Spline via SpATS," and the least desirable model is the "MovingGrid".

517 Table 6. Summary of the comparison among different models via $\mathrm{R}^{2}$, residual variance, prediction accuracy,

518 Moran's I index from data set 1. Highlighted are the most desirable values in each column.

\begin{tabular}{|c|l|c|c|c|c|c|c|}
\hline Model & $\mathbf{R}^{\mathbf{2}}$ & AIC & RSE* & $\begin{array}{c}\text { Moran's } \\
\text { I }\end{array}$ & $\begin{array}{l}\text { P-value of } \\
\text { Moran's I }\end{array}$ & $\begin{array}{l}\text { Prediction } \\
\text { Accuracy I }\end{array}$ & $\begin{array}{l}\text { Prediction } \\
\text { accuracy II }\end{array}$ \\
\hline OLS w/o RR & 0.5200 & 8978 & 1.7590 & 0.5151 & $2.2 \mathrm{e}-16$ & 0.7200 & $\mathbf{0 . 6 8 8 2}$ \\
\hline OLS w/ RR & 0.6862 & 6630 & 1.0060 & 0.5159 & $2.2 \mathrm{e}-16$ & 0.9200 & 0.6192 \\
\hline MovingGrid & 0.3409 & $\mathbf{2 6 1}$ & 1.0631 & 0.9492 & $2.2 \mathrm{e}-16$ & 0.6246 & 0.6246 \\
\hline SAR + lag & 0.6580 & 7794 & 0.8476 & 0.4575 & $2.2 \mathrm{e}-16$ & 0.8846 & $0 . .4146$ \\
\hline SAR + error & 0.6811 & 7658 & 0.8017 & 0.4599 & $2.2 \mathrm{e}-16$ & 0.8977 & 0.4279 \\
\hline SAR + mixed & 0.6780 & 7646 & 0.7771 & $\mathbf{0 . 0 3 4 9}$ & $\mathbf{0 . 0 2 1 7 9}$ & 0.9052 & 0.4422 \\
\hline ASReml AR1 & 0.7289 & $2325^{* *}$ & 0.6364 & 0.2681 & $2.2 \mathrm{e}-16$ & 0.8538 & 0.3581 \\
\hline P-Spline & $\mathbf{0 . 8 9 3 1}$ & 10065 & $\mathbf{0 . 5 5 8 2}$ & 0.0748 & $5.21 \mathrm{e}-07$ & $\mathbf{0 . 9 4 7 3}$ & 0.5124 \\
\hline
\end{tabular}

$520 *$ Residual SE: residual standard error

$521 * *$ from Asreml: AIC $=-2 *$ asreml.Obj\$loglik $+2 *$ length ( asreml.Obj\$gammas $)$

522 Prediction accuracy I: correlation coefficient between predicted and the true IDC scores of all the lines

523 Prediction accuracy II: accuracy for the lines not planted in the high IDC pressure regions 
526 Fig. 4. Heatmaps of residual values from eight models applied to data set 1 . In each of the figures,

$527 \mathrm{x}$-axis is the rows from 1 to $50 ; \mathrm{y}$-axis is the ranges 1 to 42 . The numbers under legend bars are

528 the range of residual values, and the numbers above the legend bar in the bottom half of the figure

529 are the row numbers.

530 Performance metrics and heatmaps of residuals for the eight models applied to data set 2 are

531 summarized in Table 7 and Fig. 5. From the highlighted values in Table 7, the group of SAR

532 models with either lag, error, or mixed have the best performance metrics among the eight models.

533 Based on $\mathrm{R}^{2}$, residual standard error (RSE), and Moran's I index, SAR + error term as a covariate

534 in the model ranks the best. If based on prediction accuracy, Model SAR + mixed is the best since

535 it has the highest prediction values: 0.9486 and 0.8435 for prediction accuracy I and II, respectively.

536 In contrast to the results from data set 1 , the model AR1 $\otimes$ AR1 via ASReml has the second worst

$537 \mathrm{R}^{2}$ and it is only slightly better than that of the "MovingGrid" model.

538 Table 7. Summary of the comparison among different models via $\mathrm{R}^{2}$, residual variance, prediction accuracy,

539 Moran's I index from data set 2. OLS w/o RR stands for ordinary least square without range and row; OLS

540 w/ RR for ordinary least square with range and row. Highlighted are the most desirable values in each

541 column.

\begin{tabular}{|c|l|l|l|l|l|c|c|}
\hline Model & $\mathbf{R}^{\mathbf{2}}$ & AIC & RSE & $\begin{array}{l}\text { Moran's } \\
\text { I }\end{array}$ & $\begin{array}{l}\text { P-value } \\
\text { Moran's I }\end{array}$ & $\begin{array}{l}\text { Prediction } \\
\text { Accuracy 1 }\end{array}$ & $\begin{array}{l}\text { Prediction } \\
\text { Accuracy 2 }\end{array}$ \\
\hline OLS w/o RR & 0.666 & 22657 & 1.937 & 0.2148 & $2.2 \mathrm{e}-16$ & 0.9428 & 0.8295 \\
\hline OLS w/ RR & 0.648 & 22793 & 1.923 & 0.2131 & $2.2 \mathrm{e}-16$ & 0.9429 & 0.8283 \\
\hline MovingGrid & 0.385 & $\mathbf{6 7 8 8}$ & 1.8094 & 0.8664 & $2.2 \mathrm{e}-16$ & 0.5839 & 0.7426 \\
\hline SAR + lag & 0.685 & 22521 & 1.3458 & 0.1655 & $2.2 \mathrm{e}-16$ & 0.8356 & 0.5540 \\
\hline SAR + error & $\mathbf{0 . 7 1 5}$ & 22479 & $\mathbf{1 . 3 0 8 0}$ & $\mathbf{0 . 1 5 1 8}$ & $2.2 \mathrm{e}-16$ & 0.8457 & 0.5752 \\
\hline SAR + mixed & 0.701 & 22581 & 1.3408 & 0.1778 & $2.2 \mathrm{e}-16$ & $\mathbf{0 . 9 4 8 6}$ & 0.8435 \\
\hline
\end{tabular}




\begin{tabular}{|c|c|c|c|c|c|c|c|}
\hline ASReml AR1 & 0.555 & 14689 & 1.6765 & 0.3962 & $2.2 \mathrm{e}-16$ & 0.8088 & 0.5033 \\
\hline P-Spline & 0.565 & 50678 & 1.6156 & 0.2639 & $2.2 \mathrm{e}-16$ & 0.7636 & 0.4636 \\
\hline
\end{tabular}

544 Fig 5. Heatmaps of residuals derived for each of eight models applied to data set 2. Each of the

545 images has 220 ranges by 26 rows. $\mathrm{X}$-axis is row and $\mathrm{Y}$-axis is the range. The numbers under

546 legend bars are the range of residual values, and the numbers above the legend bar in the bottom

547 half of the figure are the row numbers.

548 In contrast to application of models to data set 1 , the performance metrics from the P-spline model

549 applied to data set 2 were not the best, most of the best performance metrics were from the SAR

550 models. Based on AIC values the P-spline model has the largest AIC value 50,678, suggesting

551 that the P-spline model is overfitting the data. Three models, "OLS w/o RR," "OLS w/ RR," and

552 "SAR + mixed", have very similar prediction accuracies, 0.9428, 0.9429, and 0.9486, respectively,

553 indicating that range and row effects are not as important for making adjustements in data set 2 as

554 for data set 1 . This result is consistent with the total spatial effective dimensions $\left(\mathrm{ED}_{\mathrm{s}}\right)$ in Table 4 ,

555 in which data set 2 has a total of only $65.5 \mathrm{ED}_{\mathrm{s}}$ with a large field of 220 range by 26 rows, whereas

556 data set 1 has an $\mathrm{ED}_{\mathrm{s}}$ of 142.6 with a small field of 50 ranges by 42 rows. Heatmaps from the

557 eight models applied to data set 2 show clear spatial variation patterns (Fig 5), indicating that none

558 of the methods fully remove the spatial patterns.

559 The performance metrics from application of the eight models to data set 3 are very similar (Table

560 8) to results for data set 2 . The results of the three SAR models, "SAR + lag," "SAR + error," and

561 "SAR + mixed," are similar. The model "SAR + mixed" has the highest $\mathrm{R}^{2}(0.9491)$, prediction 
accuracy (0.9746), and smallest RSE (0.5827) (Table 8). However, in contrast to the results from data set 2 "SAR + lag" instead of model "SAR + error" has the smallest Moran's index (0.022).

564 Table 8. Summary of the comparison results among the eight models via $\mathrm{R}^{2}$, AIC, residual standard

565 errors, prediction accuracy, Moran's I index from data set 3. Highlighted are the most desirable values in 566 each column.

\begin{tabular}{|l|l|l|l|l|l|l|l|}
\hline Model & $\mathrm{R}^{2}$ & AIC & RSE & $\begin{array}{l}\text { Moran's } \\
\text { I }\end{array}$ & $\begin{array}{l}\text { P-value of } \\
\text { Moran's I }\end{array}$ & $\begin{array}{l}\text { Prediction } \\
\text { Accuracy 1 }\end{array}$ & $\begin{array}{l}\text { Prediction } \\
\text { Accuracy 2 }\end{array}$ \\
\hline OLS w/o RR & 0.8390 & 20298 & 1.8440 & 0.1456 & $2.2 \mathrm{e}-16$ & 0.9534 & 0.8020 \\
\hline OLS w/ RR & 0.8442 & 20145 & 1.8150 & 0.1358 & $2.2 \mathrm{e}-16$ & 0.9541 & 0.8033 \\
\hline MovingGrid & 0.5685 & $\mathbf{5 2 0 4}$ & 1.6790 & 0.9306 & $2.2 \mathrm{e}-16$ & 0.6569 & 0.4049 \\
\hline SAR + lag & 0.9076 & 18251 & 0.7852 & $\mathbf{0 . 0 2 2 0}$ & $\mathbf{0 . 2 3 3 0}$ & 0.9672 & 0.8471 \\
\hline SAR + error & 0.9450 & 17283 & 0.6059 & 0.0568 & $4.5 \mathrm{e}-08$ & 0.9723 & 0.8654 \\
\hline SAR + mixed & $\mathbf{0 . 9 4 9 1}$ & 17180 & $\mathbf{0 . 5 8 2 7}$ & 0.0826 & $1.8 \mathrm{e}-15$ & $\mathbf{0 . 9 7 4 6}$ & $\mathbf{0 . 8 7 5 3}$ \\
\hline ASReml AR1 & 0.6399 & 12603 & 1.5503 & 0.4233 & $2.2 \mathrm{e}-16$ & 0.8448 & 0.5004 \\
\hline B/P-Spline & 0.7494 & 41241 & 1.2935 & 0.1169 & $2.2 \mathrm{e}-16$ & 0.8684 & 0.5732 \\
\hline
\end{tabular}

568 Heatmaps of the residuals from application of the eight models to data set 3 (Fig 6) indicate that

569 models 3 and 7 produce patterns that are very similar to the heatmap of the raw IDC scores, while

570 models $4,5,6$, and 8 retain, albeit limited, spatial patterns. Comparing the legend scales, the

571 models "SAR + error" and "SAR + mixed," have the smallest residual ranges from -3 to 5, and all

572 the others have the residual ranges from -4 to +4 except that adjustments from the "MovingGrid"

573 with residual ranges from -2 to +4 . The heatmap of residuals from application of model "SAR +

574 lag," appears to have the least evidence of a spatial pattern, and Moran's I is close to 0. 
575 Fig 6. Heatmaps of residuals from data set 3 derived from eight models applied to IDC scores. The

576 numbers under legend bars are the range of residual values, and the numbers above the legend bar

577 in the bottom half of the figure are the row numbers.

578 Based on the results in Tables 6, 7, and 8 and Figures 4, 5, and 6, application of "SAR + mixed,"

579 seems to provide the best outcomes for data sets 2 and 3, and model 8, "P-Spline via SpATS,"

580 provided the best adjustements to IDC scores for data set 1.

581 With limited knowledge of the distributions of RSE associated with each model from the three

582 data sets, we chose to conduct a distribution-free nonparametric Kruskal-Wallis test to assess

583 whether there is a significant difference among RSE values generated by the eight models (Table

584 9). Model 1, "OLS w/o RR “ was the worst, and Model 1, 2, and 3 are not significantly different.

585 Similarly, model 6, "SAR + mixed" is the best, but models $4,5,6,7$, and 8 are not significantly

586 different. As shown in Fig 7, three groups were observed with models 1, 2, and 3 in group I, models

5874 , 5, and 6 in group II, and Models 7 and 8 in group III. These three groups are consistent with the

588 model's mathematical and spatial covariate structures. Pairwise t-tests among the three groups

589 show that group I and II are significantly different, with a p-value $<0.001$, Groups II and III show

590 significant differences with $p$-value $<0.01$. Group I and III are statistically different at $p$-value of

5910.1 , but not 0.05 (Table 10). 
593 Table 9. Results of the Kruskal-Wallis test.

\begin{tabular}{|c|c|c|c|}
\hline Model Name & Model No & RSE Rank & Significant tests \\
\hline OLS w/o RR & M1 & 21.67 & $\mathrm{a}$ \\
\hline OLS w/ RR & M2 & 17.67 & $\mathrm{ab}$ \\
\hline MovingGrid & M3 & 16.00 & $\mathrm{abc}$ \\
\hline ASReml AR1 & M7 & 12.00 & $\mathrm{bcd}$ \\
\hline P-Spline & M8 & 9.33 & $\mathrm{~cd}$ \\
\hline SAR + lag & M4 & 9.33 & $\mathrm{~cd}$ \\
\hline SAR + error & M5 & 7.33 & $\mathrm{~d}$ \\
\hline SAR + mixed & M6 & 6.67 & $\mathrm{~d}$ \\
\hline
\end{tabular}

595 Fig 7. Boxplot of the residual standard error (RSE) generated by application of eight spatial models

596 to three data sets consisting of range $\mathrm{x}$ row organization of plots scored for IDC. Group I consists

597 of OLS and moving grid models, group II consists of SAR models and Group III consists of

598 ASReml AR1 and P-spline models.

599

600 Table 10. Pairwise t-test of three groups of spatial models.

\begin{tabular}{|c|c|c|c|c|c|}
\hline Model group & $\begin{array}{c}\text { Mean of } \\
\mathbf{1}^{\text {st }} \text { group }\end{array}$ & $\begin{array}{c}\text { Mean of } \\
\mathbf{2}^{\text {nd }} \text { group }\end{array}$ & $\begin{array}{c}\text { Mean } \\
\text { difference }\end{array}$ & t-test & p-values \\
\hline Groups I vs II & 1.78 & 0.66 & 1.12 & -6.3367 & 0.00039 \\
\hline Groups I vs III & 1.78 & 1.42 & 0.36 & -2.0133 & 0.08396 \\
\hline Groups II vs III & 0.66 & 1.42 & -0.76 & 3.5051 & 0.00993 \\
\hline
\end{tabular}

601

602 Relative Efficiency (RE) of the spatial autoregressive (SAR) analyses. Relative to the ordinary

603 least square models without (OLS w/o RR) and with range and row information (OLS w RR)

604 efficiencies of models are presented in Table 11. The model "SAR + mixed" has the highest

605 relative efficiency of 4.21 indicating that at least four additional replicates of the OLS w/o range 
606 and row information would be needed in order to attain the same residual error. All relative

607 efficiencies from the models are larger than 1.0, demonstrating that range and row information

608 will explain significantly greater amounts of variance among IDC scores from all three data sets.

609 The last column of Table 11 shows the relative efficiency of the seven models compared with

610 Model 2, ordinary least square (OLS) with range and row in the model (OLS w/ RR). All the six

611 models with range and row, have relative efficiencies larger than 1.0. The relative efficiencies of

612 these six models indicates that there are significant improvements to be realized using more than

613 simple "range" and "row" information to correct for spatial autocorrelation for spatial variation

614 of IDC scores.

615 Table 11. Summary of relative efficiency (RE) of spatial analysis of all models compared to.

616 ordinary least square (OLS) without range and rowand ordinary least square (OLS) with range

617 and row.

\begin{tabular}{|l|c|c|}
\hline Model Name & $\begin{array}{c}\text { RE to OLS } \\
\text { w/o RR }\end{array}$ & $\begin{array}{c}\text { RE to } \\
\text { OLS w/ RR }\end{array}$ \\
\hline OLS w/o RR & 1.00 & 0.86 \\
\hline OLS w/ RR & 1.36 & 1.00 \\
\hline MovingGrid & 1.48 & 1.04 \\
\hline ASReml AR1 & 2.06 & 1.23 \\
\hline SAR + lag & 3.46 & 1.59 \\
\hline B/P-Spline & 2.55 & 1.37 \\
\hline SAR + error & 4.16 & 1.75 \\
\hline SAR + mixed & 4.21 & 1.76 \\
\hline
\end{tabular}

618

619 Lagrange Multiplier Test (LMT). Based on performance metrics and relative efficiencies the

620 Group II models provided the best outcomes from adjusting IDC scores collected from real field

621 trials (data sets 2 and 3). The Lagrange Multiplier Test (LMT) provides an overview of spatial

622 dependence by these types of models (Table 12). In order to choose within this group of models 
623 larger dependence estimates are preferred. From the dependence estimates, spatial lag and

624 spatial error models have very similar dependence estimates of 2728.1 and 2730.3, respectively.

625 Tests for possible presence of lagged variance except Lmerr, RLMerr, is 108.98. Similarly, tests

626 for possible presence of error variance (except LMlag, RLMlag), is 106.75. The last test is for

627 both error and lag model, "SAR+mixed", its dependence estimate is 2837.1 which is larger.

628 Based on LMT test results, "SAR+mixed" should be used to correct for autocorrelations.

629 However, much greater computation time is needed for SAR models. For data set 2, it took $>10$

630 hours to run the "SAR + mixed" model with 5,720 data points and a total of $\sim 25$ hours to run the

631 three data sets via a desktop Windows 10 with 16 Gb RAM and a core i7 CPU. The P-splines

632 model implemented in the SpATS R package took less than 15 minutes for all three datasets.

\section{Effects of field plot operations on spatial analyses}

634 The basic principles of experimental design are randomization, replication, and local control [74].

635 For IDC evaluations, replication and local control with an incomplete alpha-lattice design were

636 used in data sets 2 and 3. But, randomization of previously untested lines is seldom practiced.

637 Breeders usually group lines by families to ease visual evaluations. For example, a typical soybean

638 breeding program might evaluate 32 lines per family from 400 families for a total of 12,800 lines.

639 The IDC trials will be grouped into 400 trials associated with each of the families. The lines within

640 a family will be randomly assigned to plots within each trial. But the 400 trials representing

641 variability among families are usually not randomized across the field site. Rather, the families are

642 arranged by pedigree and relative maturity for purposes of visual comparisons and to accommodate

643 operational activities such as avoiding inter-plot competition between early and late maturing lines

644 and avoiding damage to plots from mechanical harvest. A harvest combine and other equipment

645 will begin with plots that are planted with early maturing families and proceed through the field 
646 from the earliest to latest maturing families. If a family is created by crossing resistant by resistant

647 lines or moderately resistant by moderately susceptible lines, there will be patches of resistant or

648 susceptible lines associated with families and these will create patterns unrelated to IDC. The

649 genotypic patterns may confound the non-genetic spatial variation pattern. Under this

650 circumstance, the spatial analysis may lead to biased adjustments. There is an opportunity to

651 develop methods that will include covariates for genotypic relationships among families and 652 expected maturities of families in the spatial models.

653 Plot size may affect spatial patterns and subsequently the effectiveness of spatial autoregressive

654 models. Soybean IDC field trials were planted in hill plots, not row-plots (Fig 8). Each hill plot

655 contained eight seeds. Hill plots were spaced 15 inches from center to center between ranges and

65610 inches from center to center between rows (left-hand image in Fig 8). In contrast plots used to

657 evaluate continuous variables such as yield and plant height are usually much larger (right-hand

658 image in Fig 8). Plot size has been shown to impact the effectiveness of spatial models [75]. Larger

659 plot sizes were better for spatial adjustements of yield values grown in heterogeneous fields.

660 However, contrary to results indicating large plot sizes are better, evidence from a 28-year case

661 study for optimizing experimental designs show that the relative efficiency of the Randomized

662 Complete Block Design increased by $240 \%$ as the length of two-row plots decreased from $5.6 \mathrm{~m}$

663 to $1.4 \mathrm{~m}$ [76]. Similar results from a comparison of different spatial models among correlated

664 error, nearest neighbor analysis, and autoregressive regression AR(1) indicates that smaller plot

665 size is more efficient to capture spatial variation and thus increase the relative efficiency of the

666 experimental design [77]. Both small plot size and ordinal IDC scores may be related to the high

667 residual standard error we found in application of the P-spline model to data sets 2 and 3. 
668 Fig 8. Images of IDC hill plots vs. yield test plots. Left image shows chlorosis phenotype of two

669 susceptible testing lines (top two hill plots) and a two resistant testing lines (bottom two hill

670 plots). Right image shows yield test plots with plants in $10 \mathrm{~m}$ rows.

\section{Inconsistent results relative to previous field plot studies.}

672 Previously, when applied to continuous traits, P-spline models have been shown to be more 673 effective and efficient than SAR models $[49,50]$. For our three data sets application of the P674 spline model was best only when applied to data set 1. For this simulated data set the P-splie model

675 identified the discontinuity boundary between iron deficient and non-deficient conditions (Fig 9,

676 bottom left-hand heatmap). Using a mixed model to obtain best linear unbiased predictions of the

677 IDC values for each of the lines, we observed a 'baseball' pattern in the heatmap (Fig 9, bottom

678 middle). Recall that the underlying genotypic values were randomly simulated based on a normal

679 distribution. Moran's index of the residual plots is 0.0748 with p-value 5.21e-07, which was much

680 smaller than the threshold p-value 0.05. Comparing the Moran's index, 0.5152 from the raw IDC

681 data, p-value less than 2.2e-16, the spatial autocorrelation coefficient is dramatically reduced, from

6820.5152 to 0.0748 , for the P-spline model. But, in terms of goodness-of-fit, there is still

683 autocorrelation left in the residual plots. There is a noticeable circle in the residual plot (Fig 9, top

684 right-hand) demonstrating that even this best model did not remove the spatial autocorrelation

685 pattern completely.

686 Fig 9. Heatmaps and histogram from application of the P-spline model to data set 1. The plots are 687 organized in 42 rows (X-axis) by 50 ranges ( $\mathrm{Y}$-axis) with a total of 2,100 plots. Genotypic BLUPs 688 are the best linear unbiased predictions of breeding values for the simulated genotypic IDC values 689 and range from -2 to +2 . 
690 Application of the P-spline model to data set 2 indicate a different spatial pattern than that from

691 the simulated data set 1 (Fig 10). Note that the heatmap of the fitted trend uses a continuous-like

692 grid, which was smoothed by 2D P-splines. The spatial surfaces displayed irregular patchy patterns

693 across field 2, and the discrepancy of the spatial trends between raw and fitted pattern remain after

694 adjustments by all eight evaluated models (Fig 5). The heat map of the residual plots has a very

695 similar spatial pattern as both fitted values and raw IDC score data, demonstrating that there is still

696 significant spatial autocorrelation after application of the P-spline model (Fig 10).

697 Fig 10. Heatmaps and histogram from data set 2. The rectangle layout consists of 26 rows (X698 axis) by 220 ranges (Y-axis) and a total of 5,720 plots. The white area in the right side of each 699 heatmap are plots without recorded data.

700 The P-spline model performed better for data set 3 (Fig 11). The fitted spatial trend matched the 701 raw field pattern very well, and heatmaps of both genotypic BLUPs and residuals appear to 702 represent randomly distributed variables. No clear noticeable spatial pattern exists like the results 703 obtaind from application to data sets 1 and 2, even though the abolute value of the Moran's I index 704 is still greater than $0(\mathrm{p}$-value $<0.05)$.

705 Fig 11. Heatmaps and histogram from data set 3 . The rectangle layout is 24 rows (X-axis) by

706220 ranges (Y-axis) with a total of 5,280 plots. The white area in the right side of each heatmap

707 are plots without data.

708 Overall results from the three residual heatmaps obtained from the P-spline model are that spatial 709 patterns or autocorrelations are not fully addressed by the model. These results are different from 710 the two previous reports in which spatial patterns had effectively been removed by 2D P-spline 711 surfaces and residuals for yield in barley [49] and sorghum [50]. We hypothesize that the 712 discrepancy between our results and those previously reported is because yield is a continuous 
713 variable and field plot variation was continuous in previously published results. Ordinal IDC

714 scores for small hill plots can be 1 (completely resistant to IDC) in one plot and it's neighboring

715 plot can be 9 (most susceptible) without a gradual transition between plots. None of the methods

716 developed to date, deal very effectively with these types of data, although the SAR methods

717 provided better RSE values.

718 Tensor product panelized splines worked very well for hybrid wheat data evaluated in Chilean and

719 Australian wheat field trials [49] as well as sorghum grain yield, and plant height [50]. However,

720 when this method was applied to soybean IDC data sets, two unexpected results were obtained: 1)

721 The effectiveness dimension analysis of the decomposed the model variables reveals that the

722 genotype or line effectiveness accounts for about $90 \%$ of the total effectiveness, while the tensor

723 product term " $\mathrm{f}(\mathrm{ROW}): \mathrm{f}(\mathrm{RANGE})$ " accounts for less than $10 \%$ of the effectiveness. 2 ) In contrast

724 to the effectiveness dimension component analysis, the variance component analysis shows that

725 tensor product term "f(ROW):f(RANGE)" accounts for over $90 \%$ of the total variance, whereas

726 genotype account for only less than 1\%. This suggests a need for development of novel methods

727 with capabilities for analysis of ordinal data obtained from fields that exhibit discontinuous

728 transitions among heterogeneous field plots. We propose that generalized linear, or possibly non-

729 linear, mixed models are most likely to solve these issues.

\section{Conclusions}

733 The effectiveness of spatial models depends on many factors, such as soil characteristics, weather

734 conditions, field plot operational activities, severity of the spatial variation, and other types of 
735 irregular patterns. From the comparison of residual standard error (RSE), $\mathrm{R}^{2}$, prediction accuracy,

736 AIC, and observation of their heatmaps generated for eight spatial models, none consistently

737 demonstrate the ability to completely remove the spatial autocorrelation for ordinal data in three

738 data sets. None-the-less, the spatial autoregressive (SAR) approach (with either lag, or error, or

739 Durbin mixed as a covariate) generated residual plots with less evidence of patterns than other

740 models. The tensor product panelized P-splines method applied to simulated data set 1, which had

741 a single circular spatial pattern performed best in this unique situation. As to the computation time

742 and user-friendliness, P-spline implemented int the R software package, SpATS, is the fastest and

743 the easiest to run. Because none of the models could consistently adjust for discontinuous 'patches'

744 of ordinal data, there is potential to develop improved novel methods that will be more effective

745 and efficient than are currently available.

\section{Conflict of Interest}

748 The authors declare that there is no conflict of interest.

750 Supplemental Data Available

751 Supplemental material is available online for this article. $\mathrm{R}$ codes and the data sets used for this

752 research are available online. 
755 This research was supported in part by the Department of Agronomy at Iowa State University, the

756 U.S. Department of Agriculture, Agricultural Research Service, project 5030-21000-062-00D.

757 Mention of trade names or commercial products in this publication is solely for the purpose of

758 providing specific information and does not imply recommendation or endorsement by the Iowa

759 State University or the U.S. Department of Agriculture. Iowa State University and USDA are equal

760 opportunity providers and employers. 
762 1. Lin SF, Baumer JS, Ivers D, de Cianzio SR, Shoemaker RC. Field and nutrient solution

763 tests measure similar mechanisms controlling iron deficiency chlorosis in soybean. Crop

764 Science. 1998;38(1):254-9. PubMed PMID: WOS:000071885100043.

$7652 . \quad$ Goos RJ, Johnson BE. A comparison of three methods for reducing iron-deficiency

766 chlorosis in soybean. Agronomy Journal. 2000;92(6):1135-9. PubMed PMID:

767 WOS:000165432000013.

768 3. YChart. US Soybeans Acres Planted [Internet web]. Web2020 [cited 2020 July 10,

769 2020]. US Soybean Acres Planted]. Available from:

770 https://ycharts.com/indicators/us soybeans acres planted.

7714 . Froehlich DM, Niebur WS, Fehr WR. Yield reduction from iron deficiency chlorosis in

772 soybeans. Agronomy Abstracts. 1980:54-5. PubMed PMID: CABI:19811608401.

773 5. Hansen NC, Schmitt MA, Anderson JE, Strock JS. iron deficiency of soybean in the

774 upper midwest and associated soil properties. Agronomy Journal. 2003;95(6):1595-601.

775 PubMed PMID: WOS:000186980300032.

776 6. Hansen NC, Jolley VD, Naeve SL, Goos RJ. iron deficiency of soybean in the north central us and associated soil properties. Soil Science and Plant Nutrition. 2004;50(7):983-7. PubMed PMID: WOS:000225652800006.

7. Niebur WS, Fehr WR. Agronomic evaluation of soybean genotypes resistant to irondeficiency chlorosis. Crop Science. 1981;21(4):551-4. PubMed PMID:

WOS:A1981ME18900018.

8. Cianzio SRd, Fehr WR, Anderson IC. Genotypic evaluation for iron deficiency chlorosis in soybeans by visual scores and chlorophyll concentration. Crop Science. 1979;19(5):644-6. PubMed PMID: CABI:19801688243.

9. Gaspar P. Management of Soybeans on Soils Prone to Iron Deficiency Chlorosis online2010 [cited 2016]. Available from:

https://www.pioneer.com/us/agronomy/iron deficiency chlorosis.htm|\#IntroductionoflronDeficie ncyChlorosis 1.

10. Spehar CR. Field screening of soya bean (glycine-max (I) merrill) germplasm for aluminum tolerance by the use of augmented design. Euphytica. 1994;76(3):203-13. doi: 10.1007/bf00022165. PubMed PMID: WOS:A1994PK33000006.

11. May KW, Kozub GC, Schaalje GB. Field-evaluation of a modified augmented design (type-2) for screening barley lines. Canadian Journal of Plant Science. 1989;69(1):9-15.

PubMed PMID: WOS:A1989T501500002.

12. Schaalje GB, Lynch DR, Kozub GC. Field-evaluation of a modified augmented design for early stage selection involving a large number of test lines without replication. Potato Research. 1987;30(1):35-45. doi: 10.1007/bf02357682. PubMed PMID: WOS:A1987H514400004.

13. Clarke GPY, Stefanova KT. Optimal design for early-generation plant-breeding trials with unreplicated or partially replicated test lines.(report). Australian \& New Zealand Journal of Statistics. 2011;53(4):461. doi: 10.1111/j.1467-842X.2011.00642.x.

801 14. Williams ER, John JA, Whitaker D. Construction of more flexible and efficient p-rep

802 designs. Australian \& New Zealand Journal of Statistics. 2014;56(1):89-96. doi:

803 10.1111/anzs.12068. PubMed PMID: WOS:000334598000007.

804 15. Moehring J, Williams ER, Piepho HP. Efficiency of augmented p-rep designs in multi-

805 environmental trials. Theoretical and Applied Genetics. 2014;127(5):1049-60. doi:

806 10.1007/s00122-014-2278-y. PubMed PMID: WOS:000336754700004. 
807

808

809

810

811

812

813

814

815

816

817

818

819

820

821

822

823

824

825

826

827

828

829

830

831

832

833

834

835

836

837

838

839

840

841

842

843

844

845

846

847

848

849

850

851

852

853

854

855

856

857

16. Williams E, Piepho HP, Whitaker D. Augmented p-rep designs. Biometrical Journal. 2011;53(1):19-27. doi: 10.1002/bimj.201000102. PubMed PMID: WOS:000287211500002. 17. Piepho HP, Buchse A, Truberg B. On the use of multiple lattice designs and alphadesigns in plant breeding trials. Plant Breeding. 2006;125(5):523-8. doi: 10.1111/j.14390523.2006.01267.x. PubMed PMID: WOS:000240440600019.

18. Yau SK. Efficiency of alpha-lattice designs in international variety yield trials of barley and wheat. Journal of Agricultural Science. 1997;128:5-9. doi: 10.1017/s0021859696003966. PubMed PMID: WOS:A1997WH64800002.

19. Anselin L, Rey SJ, SpringerLink (Online service). Perspectives on Spatial Data Analysis. Berlin, Heidelberg: Springer-Verlag Berlin Heidelberg,; 2010. Available from: http://dx.doi.org/10.1007/978-3-642-01976-0.

20. Mobley LR, Root E, Anselin L, Lozano-Gracia N, Koschinsky J. Spatial analysis of elderly access to primary care services. Int J Health Geogr. 2006;5:19. doi: 10.1186/1476-072X5-19. PubMed PMID: 16700904; PubMed Central PMCID: PMCPMC1482683.

21. Anselin L. How (not) to lie with spatial statistics. Am J Prev Med. 2006;30(2 Suppl):S3-6. doi: 10.1016/j.amepre.2005.09.015. PubMed PMID: 16458788.

22. Anselin L, Florax RJGM, Rey SJ. Advances in spatial econometrics : methodology, tools and applications. Berlin: Springer-Verlag; 2004. xxii, 513 p. p.

23. Anselin L. Spatial Econometrics. 2003. In: A companion to theoretical econometrics [Internet]. Malden, MA: Blackwell Pub.; [ 1 online resource (xvii, 709 pages)]. Available from: http://www.blackwellreference.com/subscriber/book?id=g9781405106764 9781405106764. 24. Ugrinowitsch C, Fellingam GW, Ricard MD. Limitations of ordinary least square models in analyzing repeated measures data. Medicine and Science in Sports and Exercise. 2004;36(12):2144-8. doi: 10.1249/01.MSS.0000147580.40591.75.

25. Clarke FR, Baker RJ, Depauw RM. Moving mean and least-squares smoothing for analysis of grain-yield data. Crop Science. 1994;34(6):1479-83. PubMed PMID: WOS:A1994PR29900011.

26. Rosielle AA. Comparison of lattice designs, check plots, and moving means in wheat breeding trials. Euphytica. 1980;29(1):129-33. doi: 10.1007/bf00037258. PubMed PMID: WOS:A1980JV21600017.

27. Townleysmith TF, Hurd EA. Use of moving means in wheat yield trials. Canadian Journal of Plant Science. 1973;53(3):447-50. PubMed PMID: WOS:A1973Q997900003. 28. Diers BW, Voss BK, Fehr WR. Moving-mean analysis of field-tests for iron efficiency of soybean. Crop Science. 1991;31(1):54-6. PubMed PMID: WOS:A1991EY83200013.

29. Mak C, Harvey BL, Berdahl JD. Evaluation of control plots and moving means for error control in barley nurseries. Crop Science. 1978;18(5):870-3. PubMed PMID:

WOS:A1978FW50200049.

30. Technow F. R package mvngGrAd: moving grid adjustment in plant breeding field trials. R package version 015. 2015:1-12.

31. Dormann E, Wokrina T. Anisotropy and spatial restriction of conduction electron diffusion in perylene radical cation salt. Synthetic Metals. 1997;86(1-3):2183-4. doi: 10.1016/s03796779(97)81087-9. PubMed PMID: WOS:A1997WZ98800190.

32. Lado B, Matus I, Rodriguez A, Inostroza L, Poland J, Belzile F, et al. Increased Genomic Prediction Accuracy in Wheat Breeding Through Spatial Adjustment of Field Trial Data. G3Genes Genomes Genetics. 2013;3(12):2105-14. doi: 10.1534/g3.113.007807. PubMed PMID: WOS:000328334500001.

33. Tobler WR. Smooth pycnophylactic interpolation for geographical regions. J Am Stat Assoc. 1979;74(367):519-30. Epub 1979/01/01. doi: 10.1080/01621459.1979.10481647. PubMed PMID: 12310706.

34. Anselin L. Spatial Econometrics. In: Baltagi BH, editor. A companion to theoretical econometrics. Malden, Mass.: Blackwell; 2001. p. xviii, 709 p. 
858

859

860

861

862

863

864

865

866

867

868

869

870

871

872

873

874

875

876

877

878

879

880

881

882

883

884

885

886

887

888

889

890

891

892

893

894

895

896

897

898

899

900

901

902

903

904

905

906

907

35. Dormann CF. Effects of incorporating spatial autocorrelation into the analysis of species distribution data. Global Ecology and Biogeography. 2007;16(2):129-38. doi: 10.1111/j.14668238.2006.00279.x. PubMed PMID: WOS:000244274900001.

36. Gleeson AC, Cullis BR. Residual maximum-likelihood (reml) estimation of a neighbor model for field experiments. Biometrics. 1987;43(2):277-88. doi: 10.2307/2531812. PubMed PMID: WOS:A1987H846200002.

37. Cullis BR, Gleeson AC. Spatial-analysis of field experiments - an extension to 2 dimensions. Biometrics. 1991;47(4):1449-60. doi: 10.2307/2532398. PubMed PMID: WOS:A1991GY51200018.

38. Hu XY, Spilke J. Comparison of various spatial models for the analysis of cultivar trials. New Zealand Journal of Agricultural Research. 2009;52(3):277-87. PubMed PMID: WOS:000271885700005.

39. Wilkinson GN, Eckert SR, Hancock TW, Mayo O. NEAREST NEIGHBOR (NN) ANALYSIS OF FIELD EXPERIMENTS. Journal of the Royal Statistical Society Series BStatistical Methodology. 1983;45(2):151-211. PubMed PMID: WOS:A1983RL82000001. 40. Piepho HP, Richter C, Williams E. Nearest neighbour adjustment and linear variance models in plant breeding trials. Biometrical Journal. 2008;50(2):164-89. doi: 10.1002/bimj.200710414. PubMed PMID: WOS:000255262700002.

41. Ainsley AE, Dyke GV, Jenkyn JF. Inter-plot interference and nearest-neighbor analysis of field experiments. Journal of Agricultural Science. 1995;125:1-9. PubMed PMID: WOS:A1995RN67100001.

42. Federer WT. Recovery of interblock, intergradient, and intervariety information in incomplete block and lattice rectangle designed experiments. Biometrics. 1998;54(2):471-81. doi: 10.2307/3109756. PubMed PMID: WOS:000074161600006.

43. Kempton R, Mead R, Engel B, ter Braak CJF, Nelder JA, Morton R, et al. The analysis of designed experiments and longitudinal data by using smoothing splines - Discussion. Journal of the Royal Statistical Society Series C-Applied Statistics. 1999;48:300-11. PubMed PMID: WOS:000080769400002.

44. Gilmour A, R, Cullis B, Verbyla AP. Accounting for Natural and Extraneous Variation in the Analysis of Field Experiments Journal of Agricultural, Biological, and Environmental Statistics 1997;2(3):269-93.

45. Stefanova KT, Smith AB, Cullis BR. Enhanced Diagnostics for the Spatial Analysis of Field Trials. Journal of Agricultural Biological and Environmental Statistics. 2009;14(4):392-410. doi: 10.1198/jabes.2009.07098. PubMed PMID: WOS:000272634200002.

46. Dhrymes P. Introductory Econometrics by Phoebus Dhrymes. 1st ed. 2017.. ed: Cham : Springer International Publishing : Imprint: Springer; 2017.

47. Cappa EP, Cantet RJC. Bayesian estimation of a surface to account for a spatial trend using penalized splines in an individual-tree mixed model. Canadian Journal of Forest Research-Revue Canadienne De Recherche Forestiere. 2007;37(12):2677-88. doi: 10.1139/x07-116. PubMed PMID: WOS:000253179200024.

48. Cappa EP, Lstiburek M, Yanchuk AD, El-Kassaby YA. Two-dimensional penalized splines via Gibbs sampling to account for spatial variability in forest genetic trials with small amount of information available. Silvae Genetica. 2011;60(1):25-35. PubMed PMID: WOS:000289329900004.

49. Rodríguez-Álvarez MX, Boer MP, van Eeuwijk FA, Eilers PHC. Spatial Models for Field Trials. 2016.

50. Velazco JG, Rodriguez-Alvarez MX, Boer MP, Jordan DR, Eilers PHC, Malosetti M, et al. Modelling spatial trends in sorghum breeding field trials using a two-dimensional P-spline mixed model. Theor Appl Genet. 2017;130(7):1375-92. doi: 10.1007/s00122-017-2894-4. PubMed PMID: 28374049; PubMed Central PMCID: PMCPMC5487705. 
908

909

910

911

912

913

914

915

916

917

918

919

920

921

922

923

924

925

926

927

928

929

930

931

932

933

934

935

936

937

938

939

940

941

942

943

944

945

946

947

948

949

950

951

952

953

954

955

956

957

51. Lado B, Matus I, Rodriguez A, Inostroza L, Poland J, Belzile F, et al. Increased genomic prediction accuracy in wheat breeding through spatial adjustment of field trial data. G3 (Bethesda). 2013;3(12):2105-14. doi: 10.1534/g3.113.007807. PubMed PMID: 24082033; PubMed Central PMCID: PMCPMC3852373.

52. Frank E. Harrell J. Regression Modeling Strategies: Springer; 2nd ed. 2015 edition (August 15, 2015); 2015. 582 p.

53. Technow F. R package mvngGrAd: moving grid adjustment in plant breeding field trials. 2015.

54. Bivand RS. Applied Spatial Data Analysis with R by Roger S. Bivand, Edzer Pebesma, Virgilio Gómez-Rubio. 2nd ed.. ed. Pebesma E, Gómez-Rubio V, editors: New York, NY : Springer New York : Imprint: Springer; 2013.

55. Bivand R, Piras G. Comparing Implementations of Estimation Methods for Spatial Econometrics. J Stat Soft. 2015;63(18). doi: 10.18637/jss.v063.i18.

56. Butler DG, Cullis BR, Gilmour AR, Gogel BG, Thompson R. ASReml-R Reference Manual Version 4: VSN International Ltd, Hemel Hempstead, HP1 1ES, UK.; 2017. Available from: https://asreml.kb.vsni.co.uk/wp-content/uploads/sites/3/2018/02/ASReml-R-ReferenceManual-4.pdf.

57. Rodríguez-Álvarez M. Correcting for spatial heterogeneity in plant breeding experiments with P-splines. Spatial Statistics. 2018;23:52-71. doi: 10.1016/j.spasta.2017.10.003.

58. Technow F. R package mvngGrAd: Moving grid adjustment in plant breeding field trials. R package. 2015;2.

59. Bivand R. Implementing Spatial Data Analysis Software Tools in R. Geographical Analysis. 2006;38(1):23-40. doi: 10.1111/j.0016-7363.2005.00672.x.

60. Baltagi BH, Liu L. Testing for random effects and spatial lag dependence in panel data models. Statistics and Probability Letters. 2008;78(18):3304-6. doi: 10.1016/j.spl.2008.06.014. 61. Anselin L, Moreno R. Properties of tests for spatial error components. Regional Science and Urban Economics. 2003;33(5):595-618. doi: 10.1016/S0166-0462(03)00008-5.

62. Bekti R. Sutikno. Spatial Durbin model to identify influential factors of diarrhea. J Math Stat. 2012;8:396-402.

63. Dormann CF, McPherson JM, Araújo MB, Bivand R, Bolliger J, Carl G, et al. Methods to Account for Spatial Autocorrelation in the Analysis of Species Distributional Data: A Review. Ecography. 2007;30(5):609-28. doi: 10.1111/j.2007.0906-7590.05171.x.

64. Butler DG, Cullis BR, Gilmour AR, Gogel BJ. mixed models for S language environments ASReml-R reference manual2009.

65. Velazco JG, Rodríguez-Álvarez MX, Boer MP, Jordan DR, Eilers PHC, Malosetti M, et al. Modelling spatial trends in sorghum breeding field trials using a two-dimensional P-spline mixed model. TAG Theoretical and applied genetics Theoretische und angewandte Genetik. 2017;130(7):1375. doi: 10.1007/s00122-017-2894-4.

66. Lee DJ, Durban M, Eilers P. Efficient two-dimensional smoothing with P-spline ANOVA mixed models and nested bases. Computational Statistics \& Data Analysis. 2013;61:22-37. doi: 10.1016/j.csda.2012.11.013. PubMed PMID: WOS:000315552600003.

67. Ebeling H, White DA, Rangarajan FVN. ASMOOTH: a simple and efficient algorithm for adaptive kernel smoothing of two-dimensional imaging data. Monthly Notices of the Royal Astronomical Society. 2006;368(1):65-73. doi: 10.1111/j.1365-2966.2006.10135.x. PubMed PMID: WOS:000236797600022.

68. James G. An Introduction to Statistical Learning with Applications in R / by Gareth James, Daniela Witten, Trevor Hastie, Robert Tibshirani. 1st ed. 2013.. ed. Witten D, Hastie T, Tibshirani R, editors: New York, NY : Springer New York : Imprint: Springer; 2013.

69. Fortin M-J, Dale MRT. Spatial autocorrelation in ecological studies: a legacy of solutions and myths. Geographical Analysis. 2009;41(4):392. doi: 10.1111/j.1538-4632.2009.00766.x. 
70. Bivand R, Müller WG, Reder M. Power calculations for global and local Moran's I. Computational Statistics and Data Analysis. 2009;53(8):2859-72. doi: 10.1016/j.csda.2008.07.021.

71. Clewer AG. Practical statistics and experimental design for plant and crop science / Alan G. Clewer and David H. Scarisbrick. Scarisbrick DH, editor. Chichester, New York: Chichester, New York : J. Wiley; 2001.

964 72. Nychka D. Tools for Spatial Data. 2016. doi: 10.5065/D6W957CT.

965 73. Abd El-Mohsen AA, Abo-Hegazy SRE. Comparing the Relative Efficiency of Two

966 Experimental Designs in Wheat Field Trials. EJPB. 2013;17(1):1-17. doi: 10.12816/0003974.

967 74. Mead R, Gilmour SG, Mead A. Statistical Principles for the Design of Experiments

968 Introduction. Statistical Principles for the Design of Experiments. 2012;(36):3-8. PubMed PMID:

969 WOS:000315678500002.

$97075 . \quad K n o r z e r ~ H$, Hartung K, Piepho HP, Lewandowski I. Assessment of variability in biomass

971 yield and quality: what is an adequate size of sampling area for miscanthus? Global Change

972 Biology Bioenergy. 2013;5(5):572-9. doi: 10.1111/gcbb.12027. PubMed PMID:

973 WOS:000323200200009.

974 76. Casler MD. Finding Hidden Treasure: A 28-Year Case Study for Optimizing

975 Experimental Designs. Communications in biometry and crop science 2013;8(1):23-8.

976 77. Sripathi R, Conaghan P, Grogan D, Casler MD. Spatial Variability Effects on Precision

977 and Power of Forage Yield Estimation. Crop Science. 2017;57(3):1383-93. doi:

978 10.2135/cropsci2016.08.0645. PubMed PMID: WOS:000404483500029.

\section{Supporting information}

980 Supplemental Fig 1. IDC field variation heatmap and patterns. (A) Four IDC field variation patterns from

981 heatmaps corresponding to three regions with IDC. (B) Simulated data set 1 with circle pattern. (C) IDC

982 testing field with IDC spatial variability. (D) The three types of the testing locations clustered by soil

983 properties and displayed relative to the first two pricipal components. 

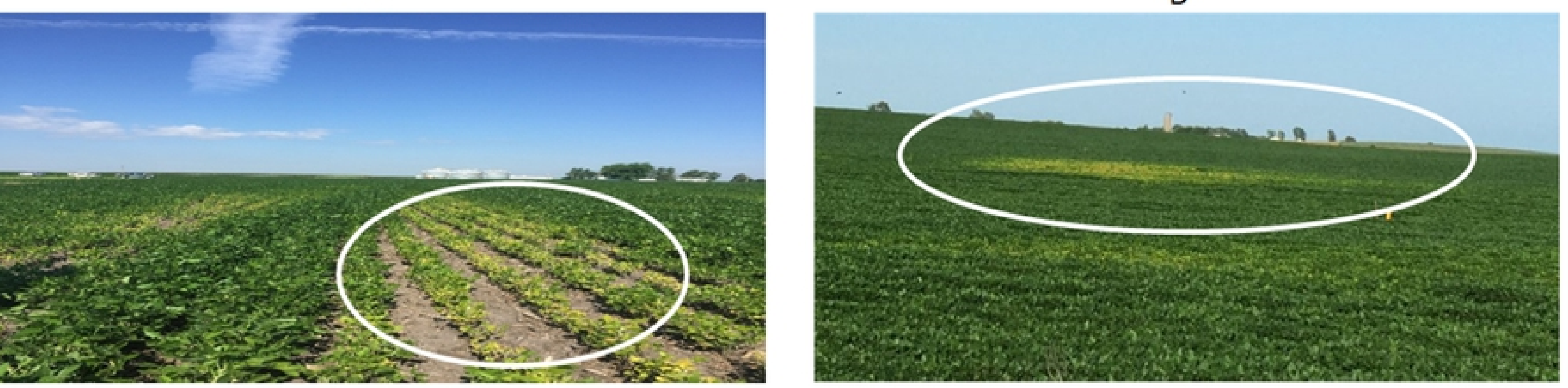

Figure 


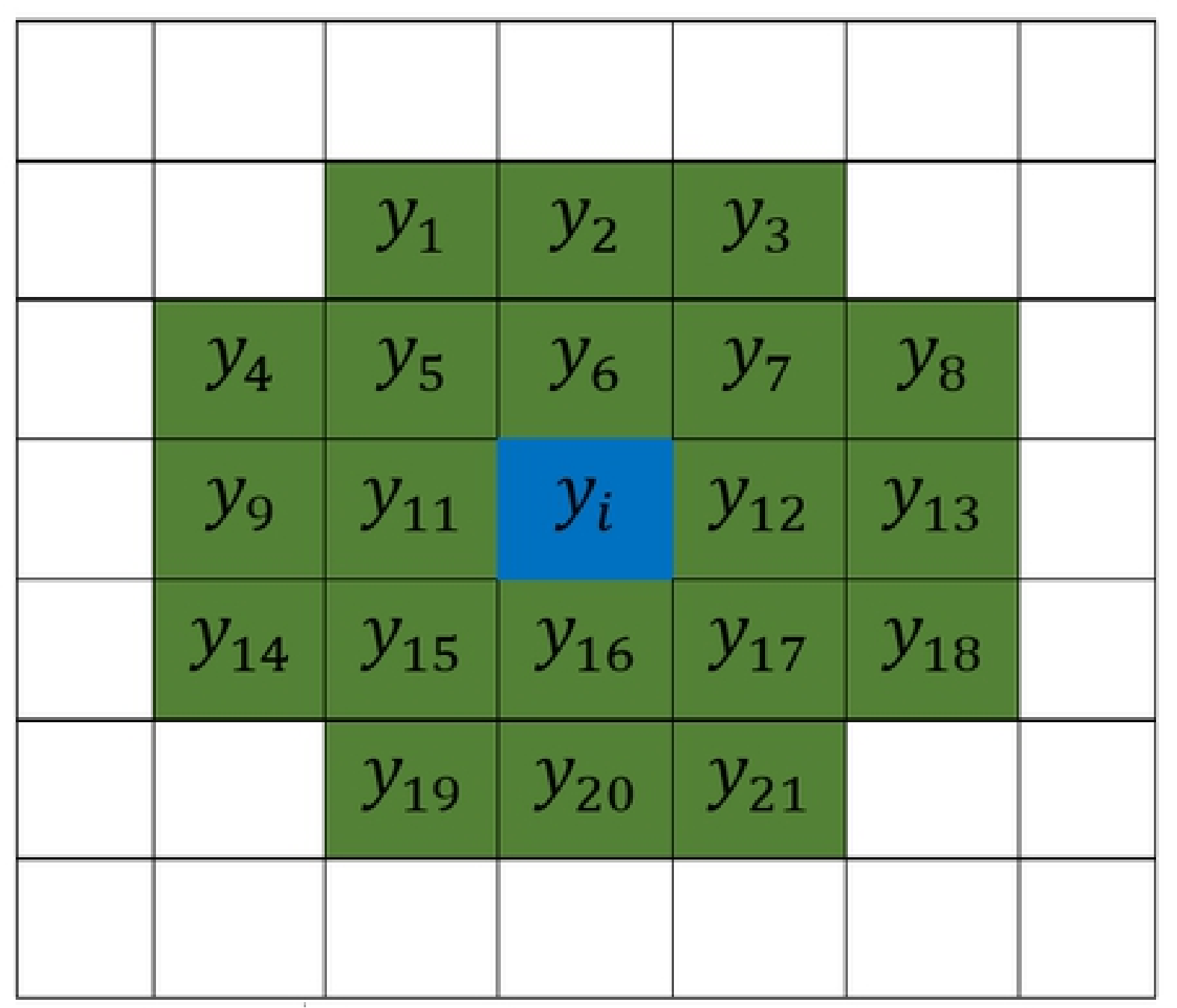

Figure 
Model 1: OLS w/o range and row
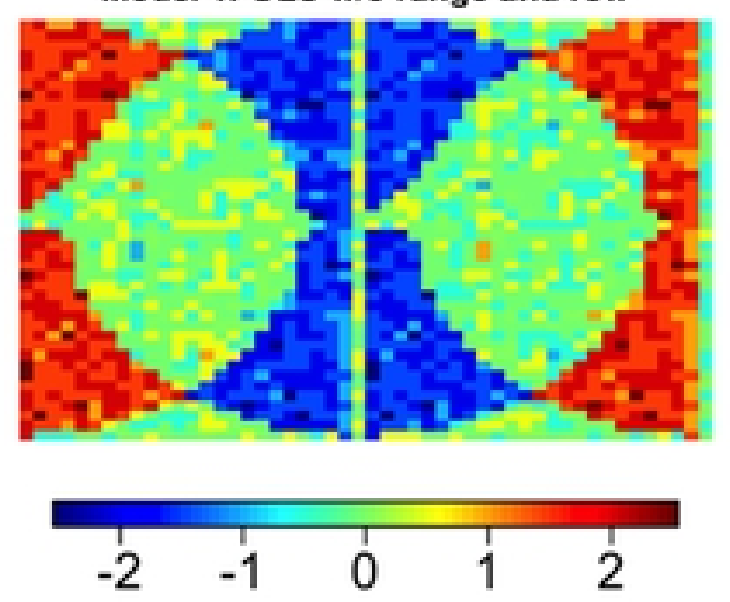

Model 4: Spatial Autoregreesive + Lag
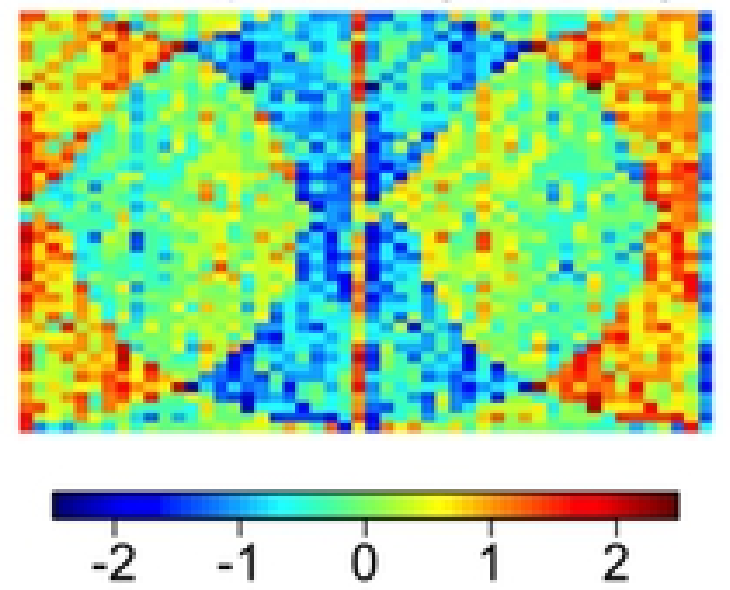

Model 7: Spatial Autoregreesive via AsremI_R

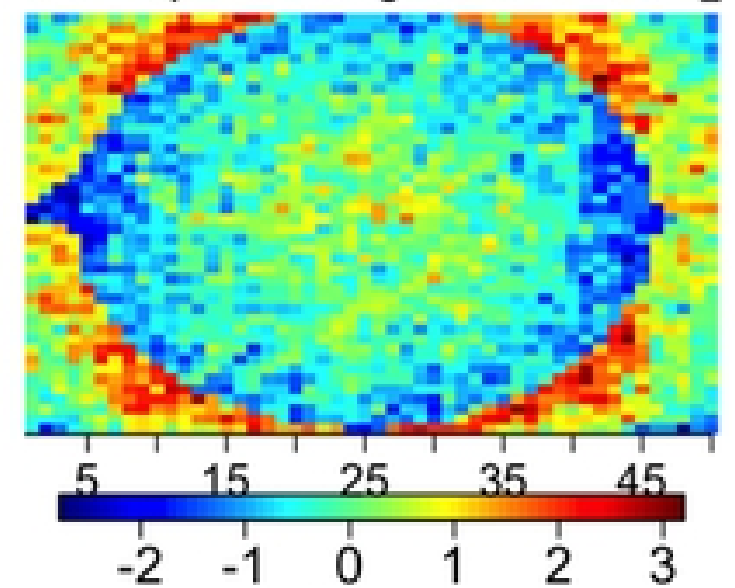

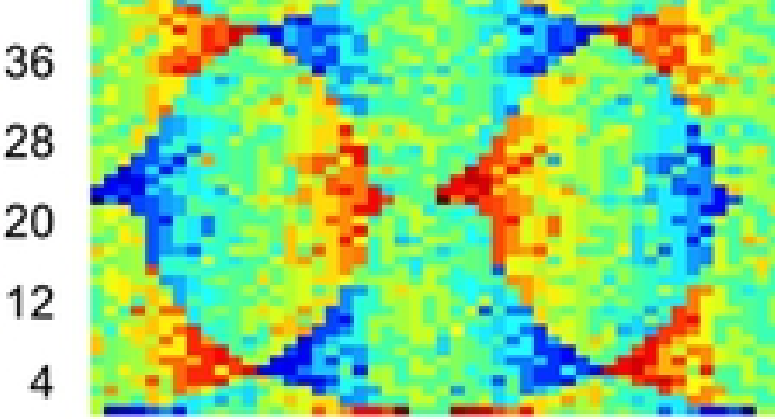

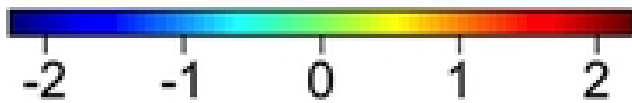

Model 5: Spatial Autoregreesive + error
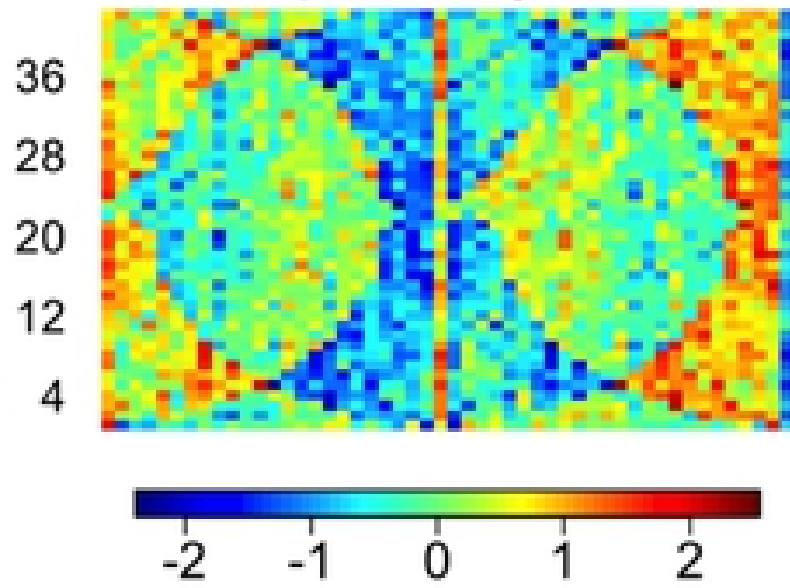

Model : B/P-Splines via SpATS

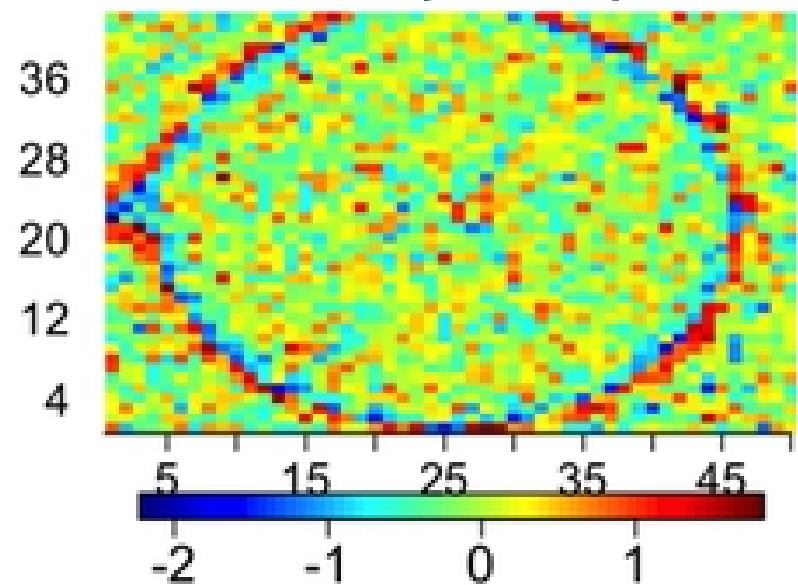

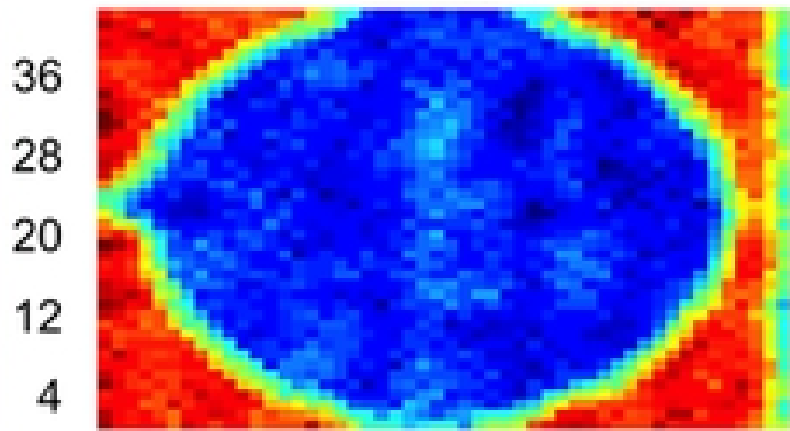

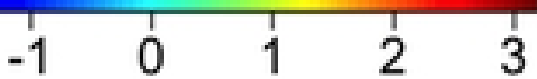

Model 6: Spatial Autoregreesive + mixed
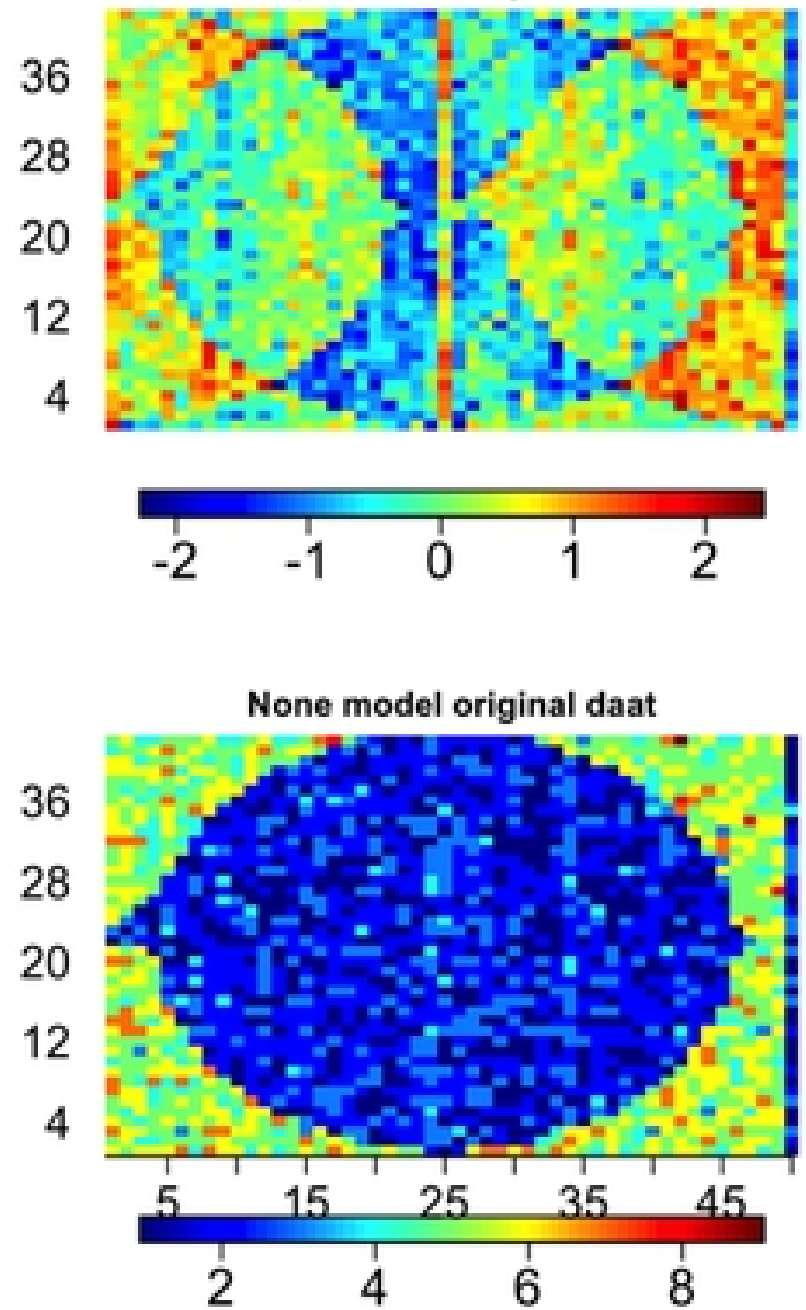

Figure 

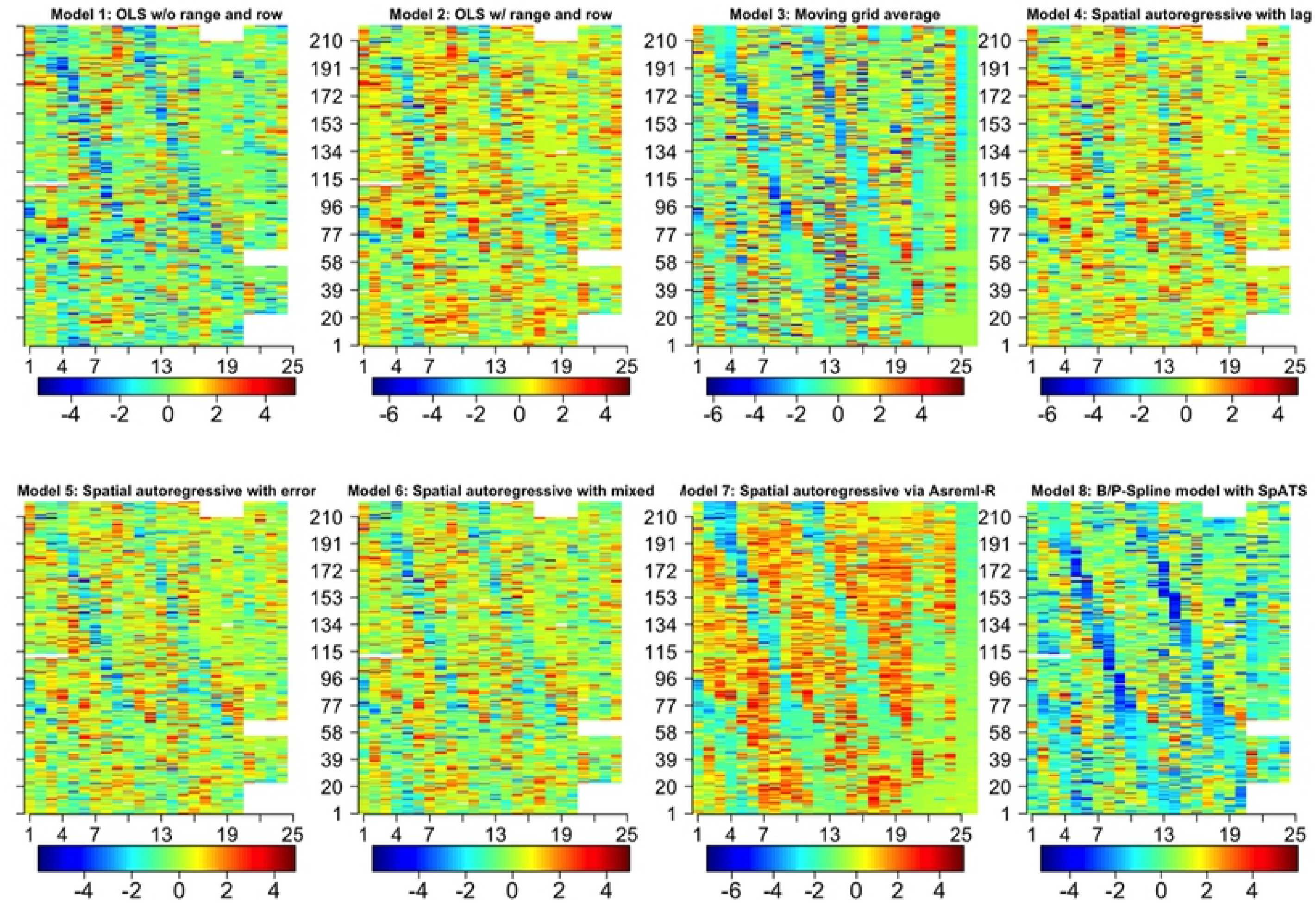

Model 8: B/P-Spline model with SpATS

Figure

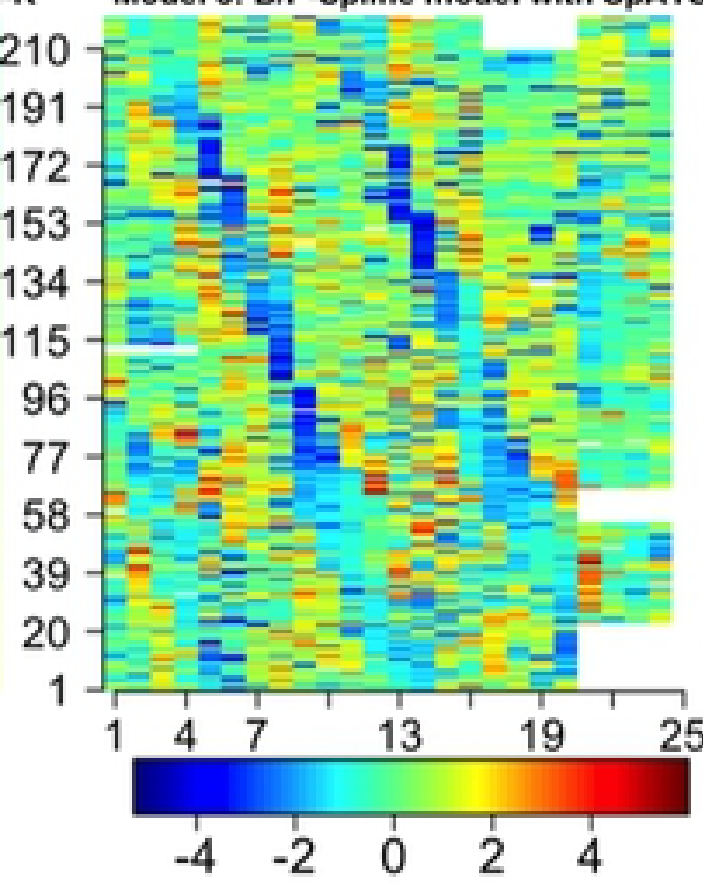




\section{Group I}

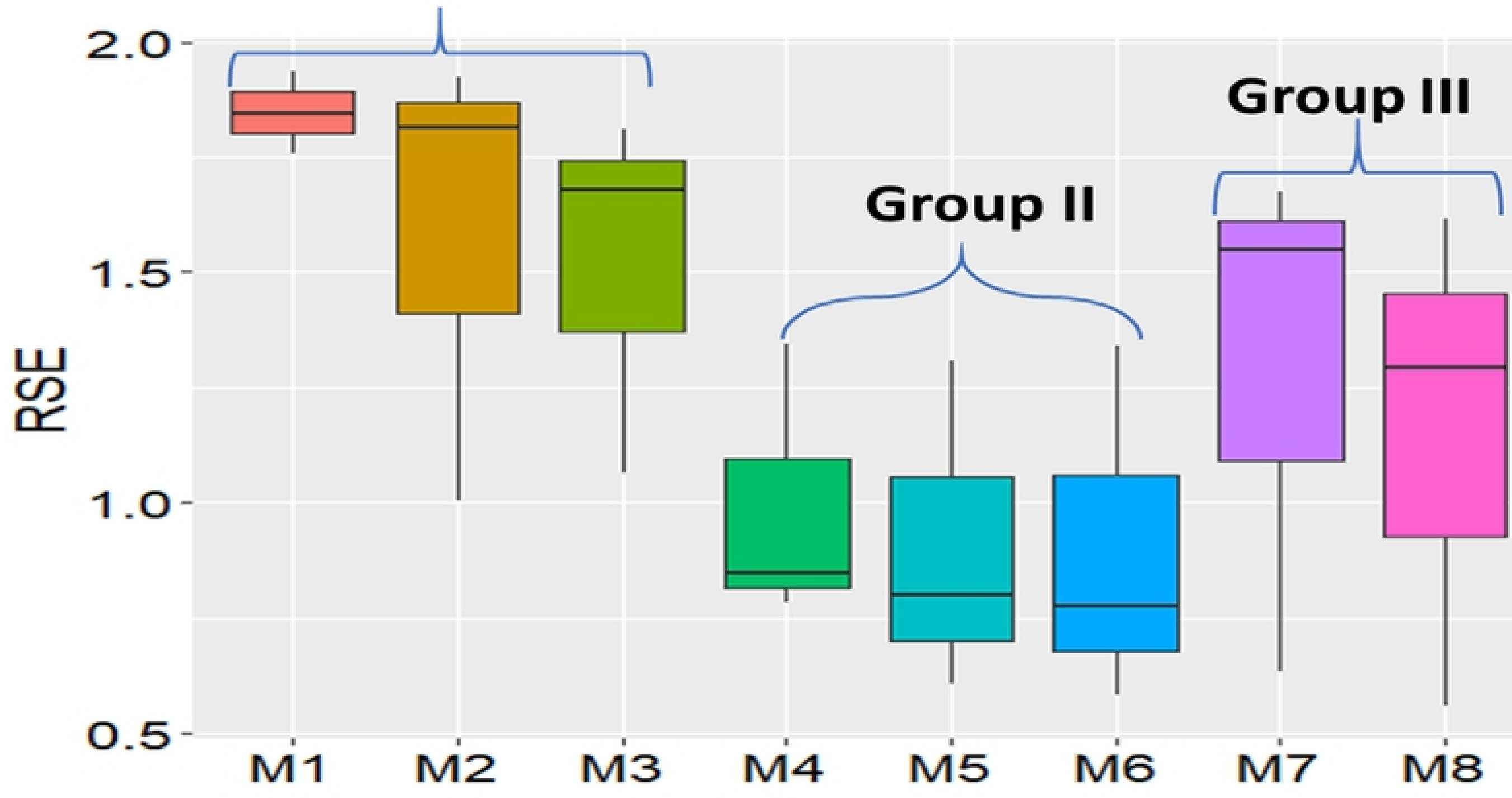

Figure 
A

B
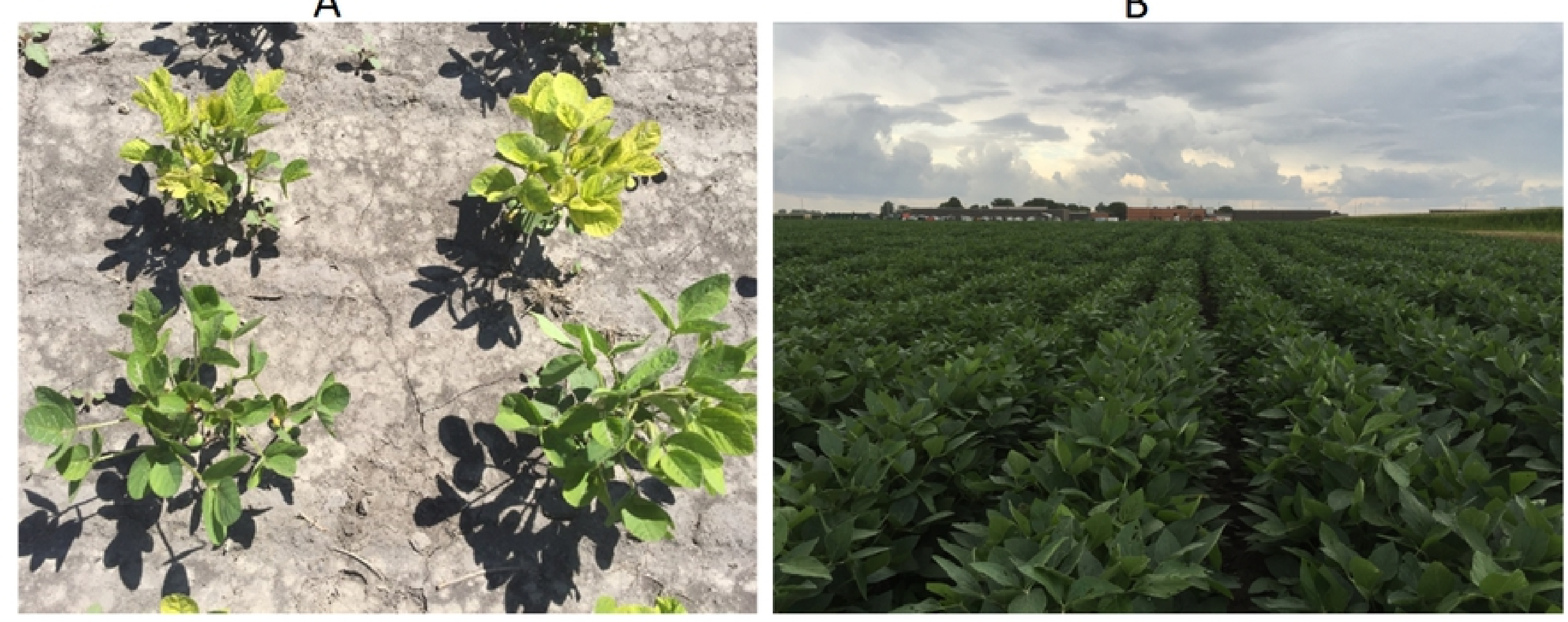

Figure 

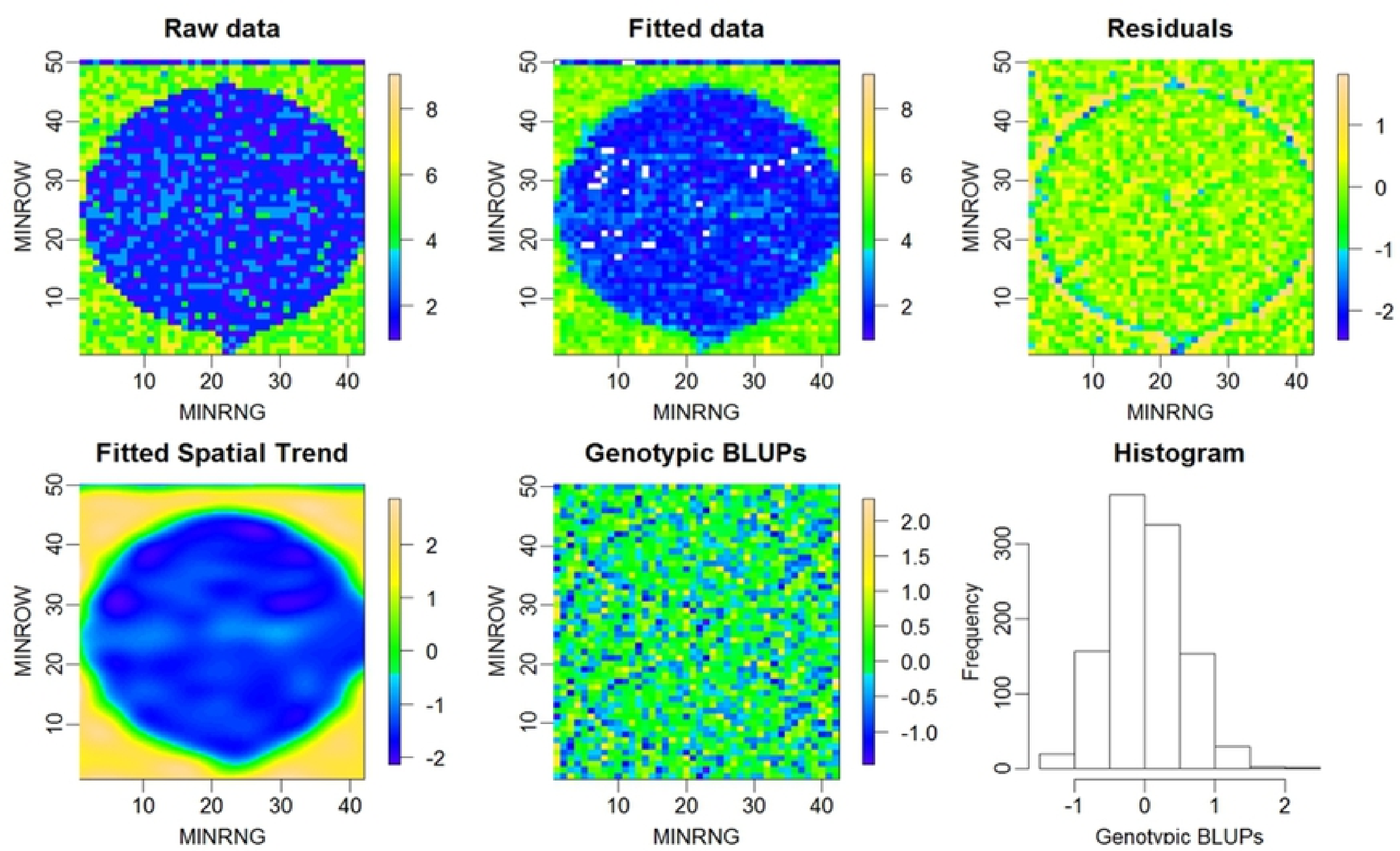

Figure 
Raw data

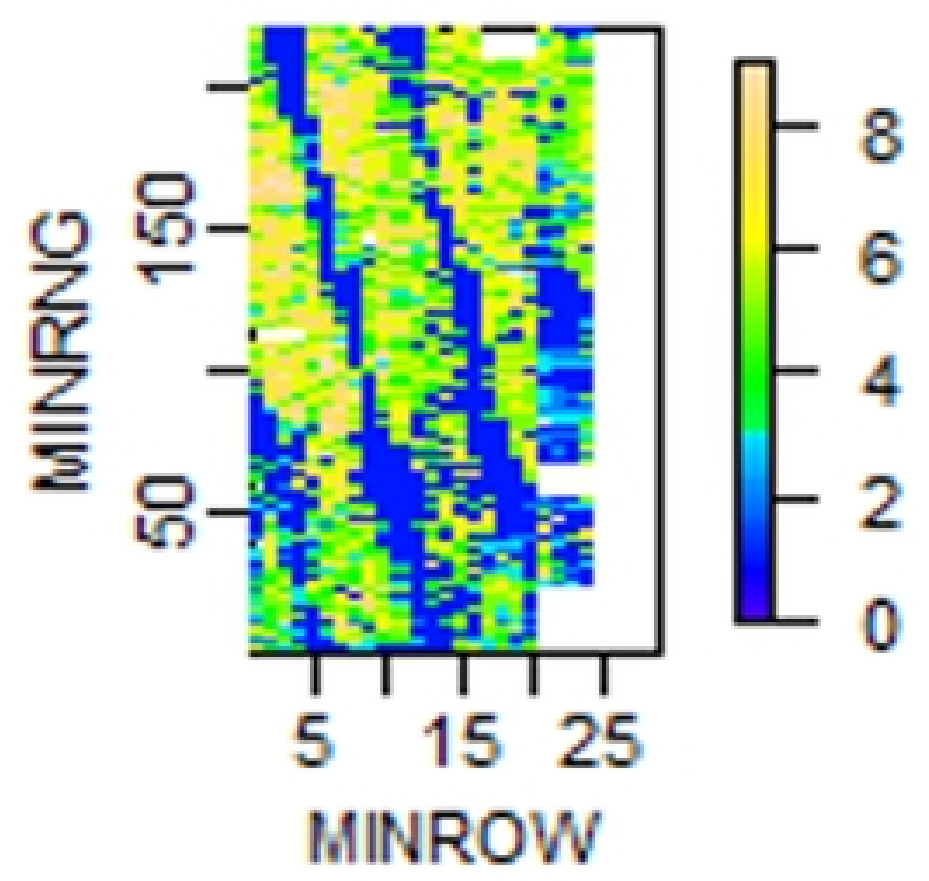

Fitted Spatial Trend

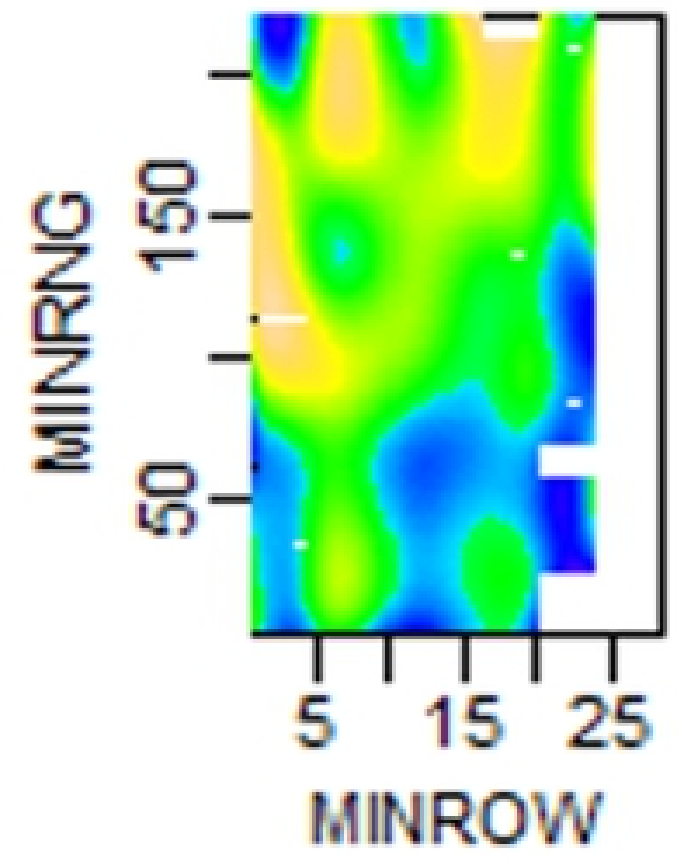

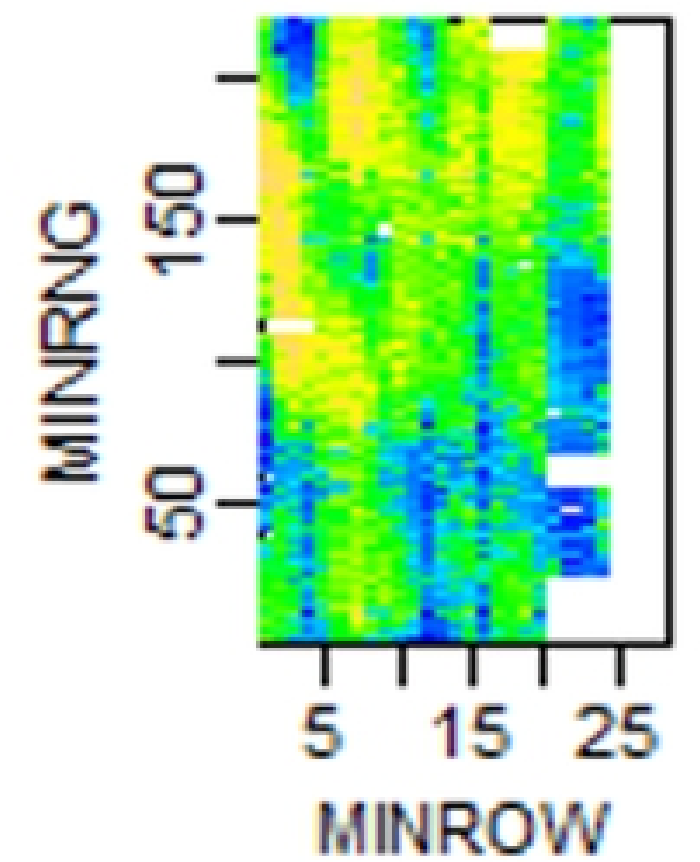

Genotypic BLUPs
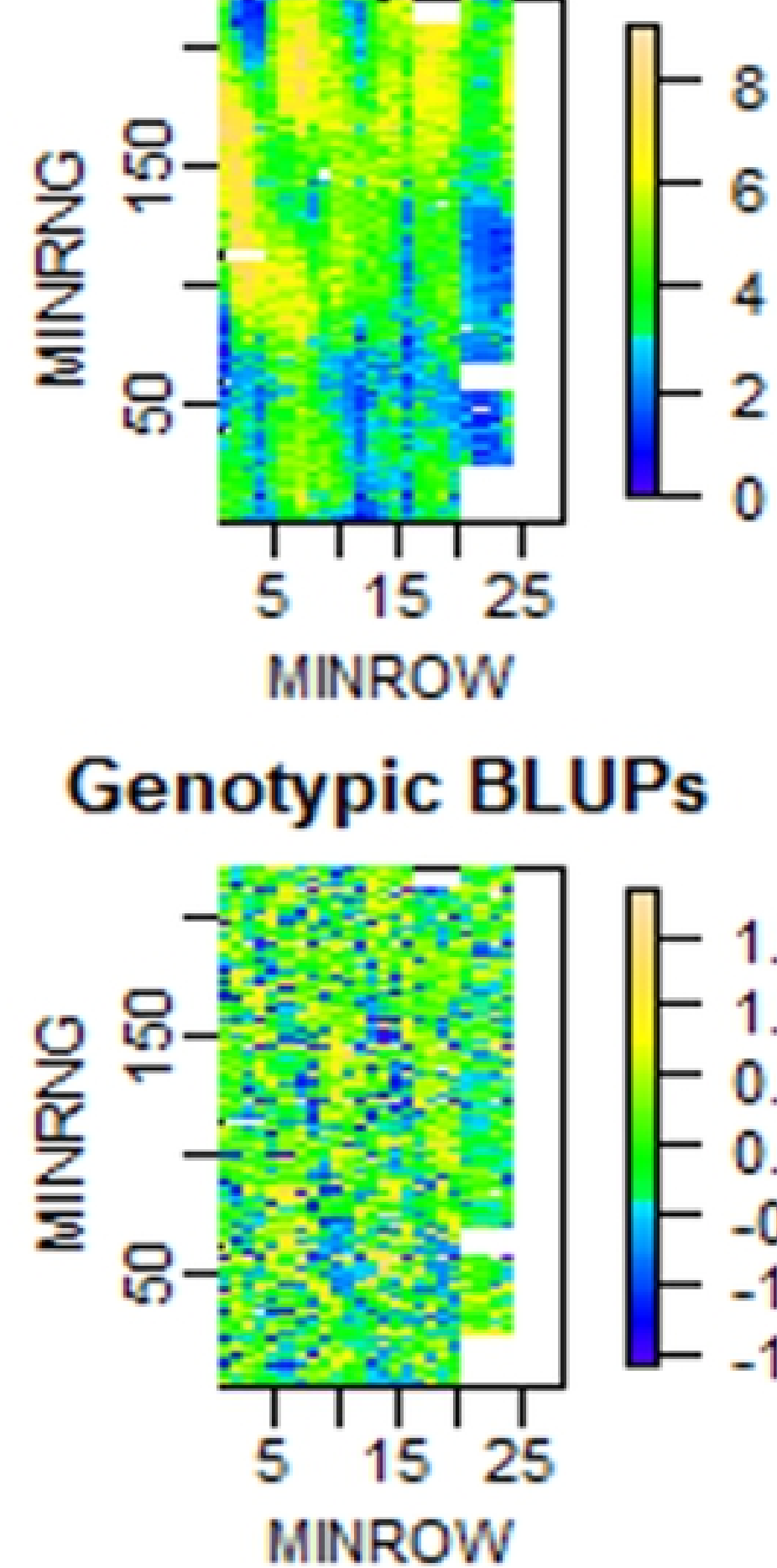

Residuals

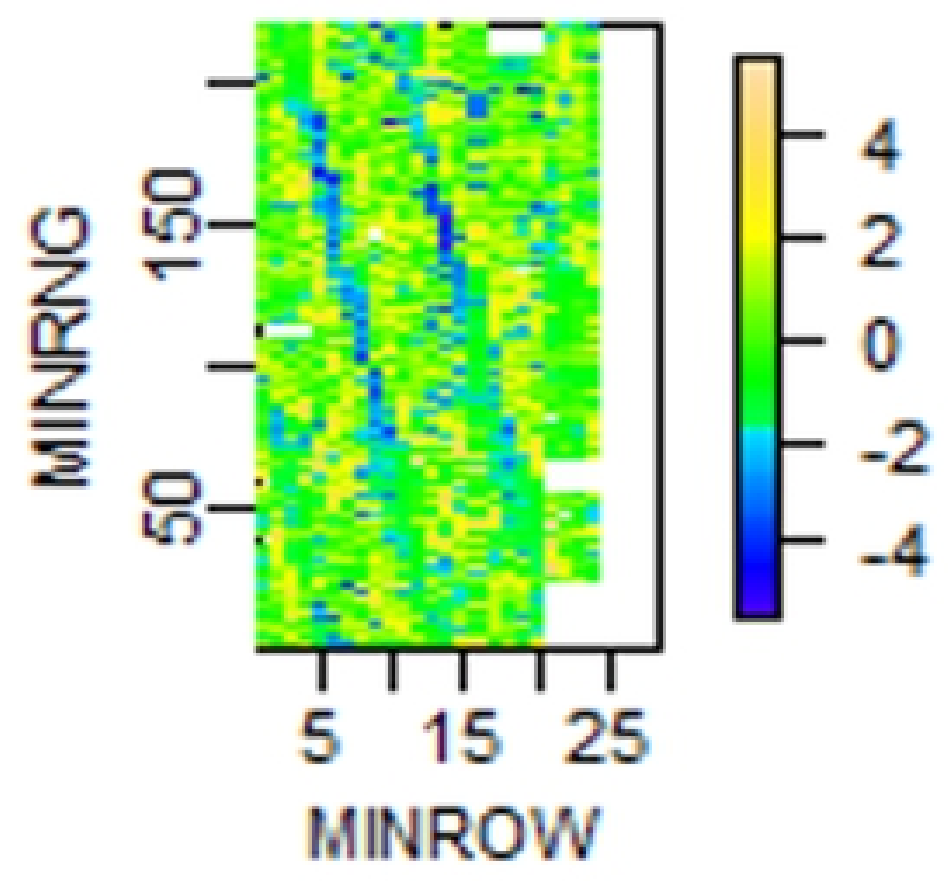

Histogram
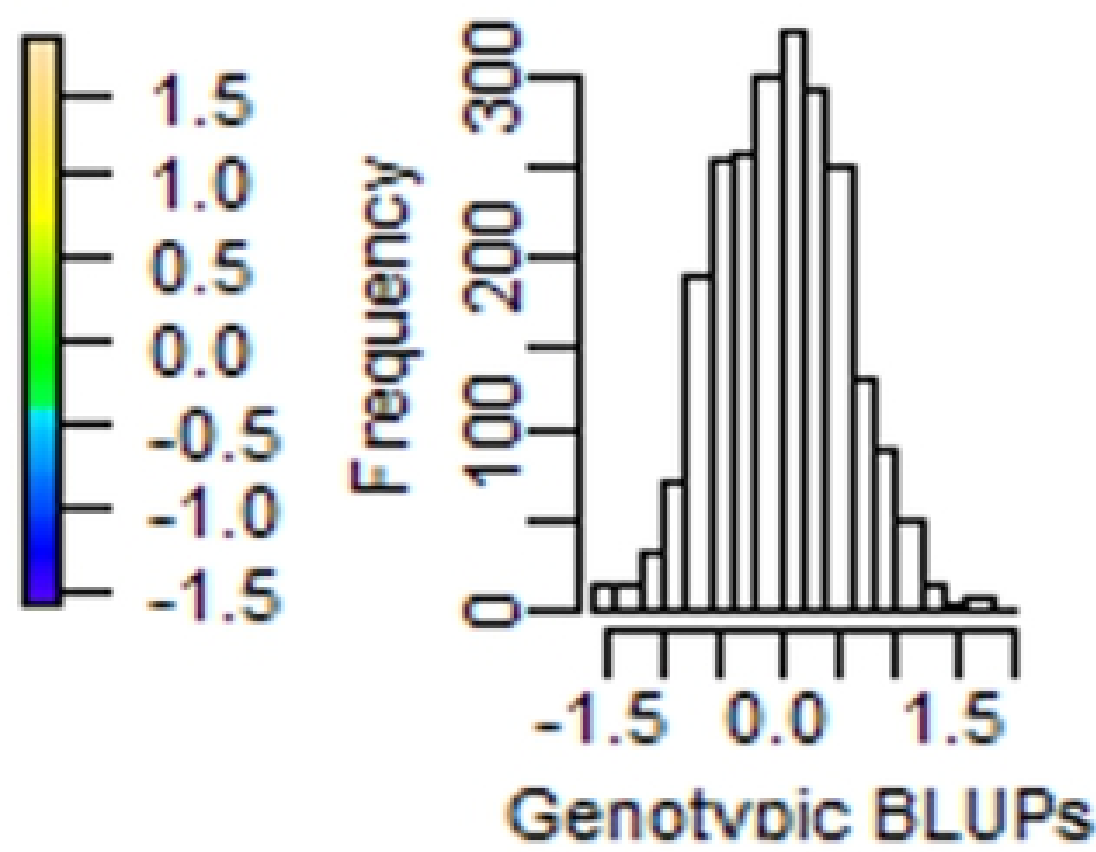

Figure 
Raw data

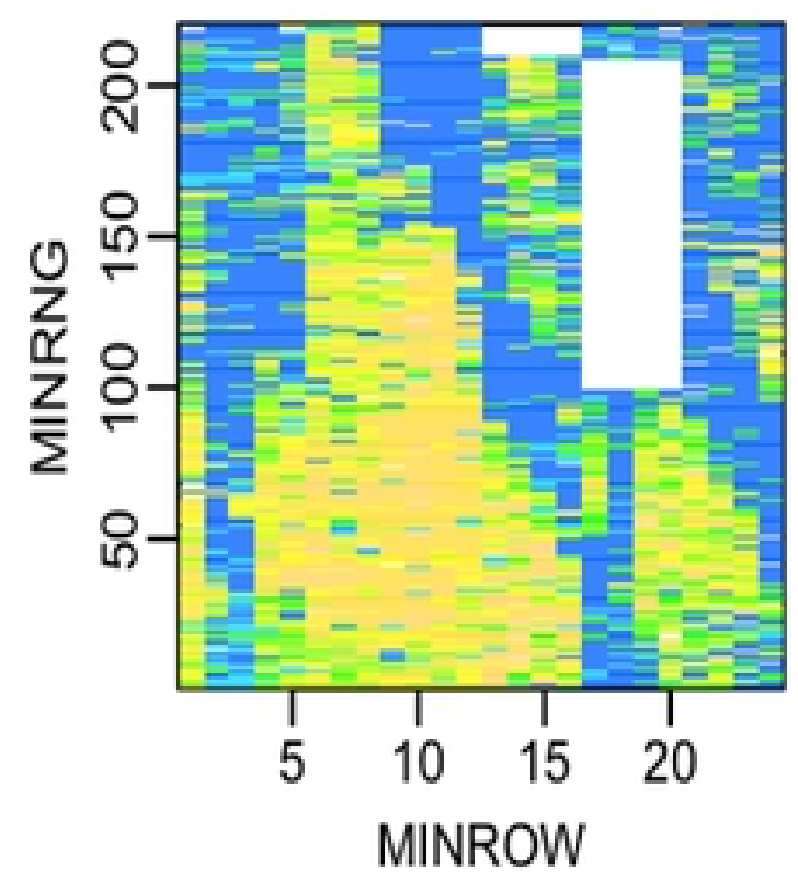

Fitted Spatial Trend

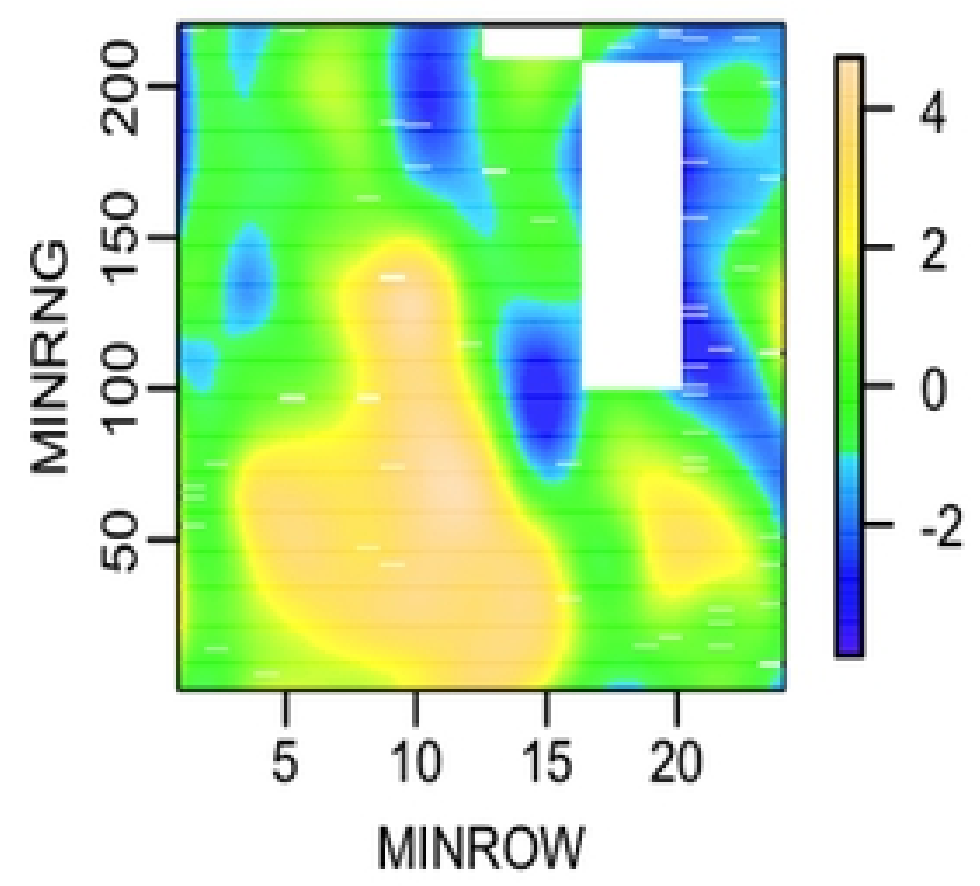

Fitted data

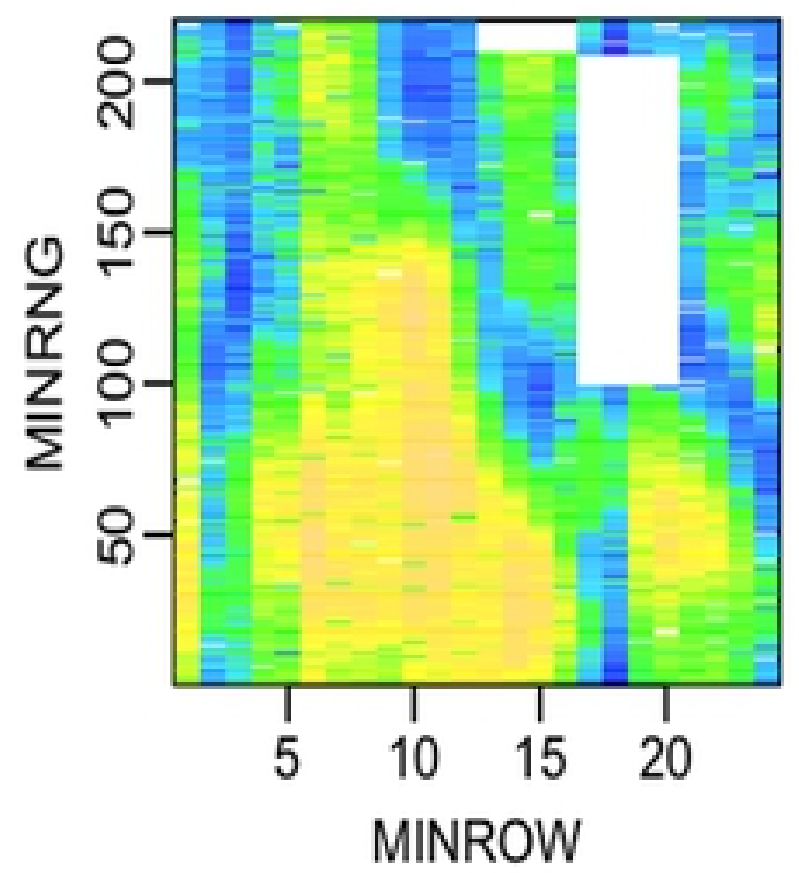

Genotypic BLUPs

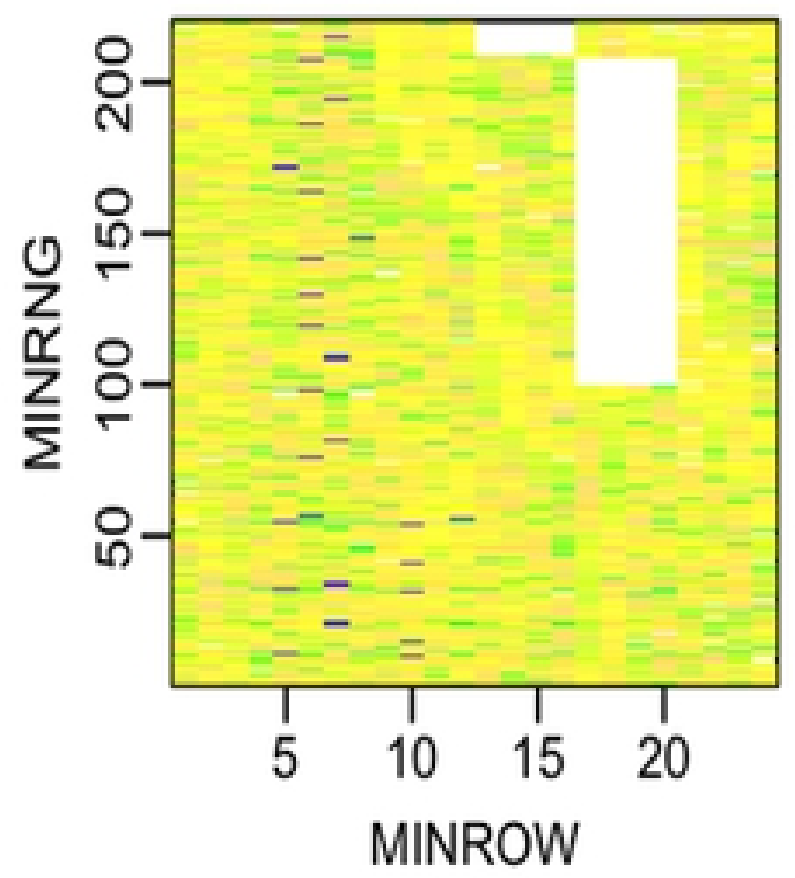

Residuals

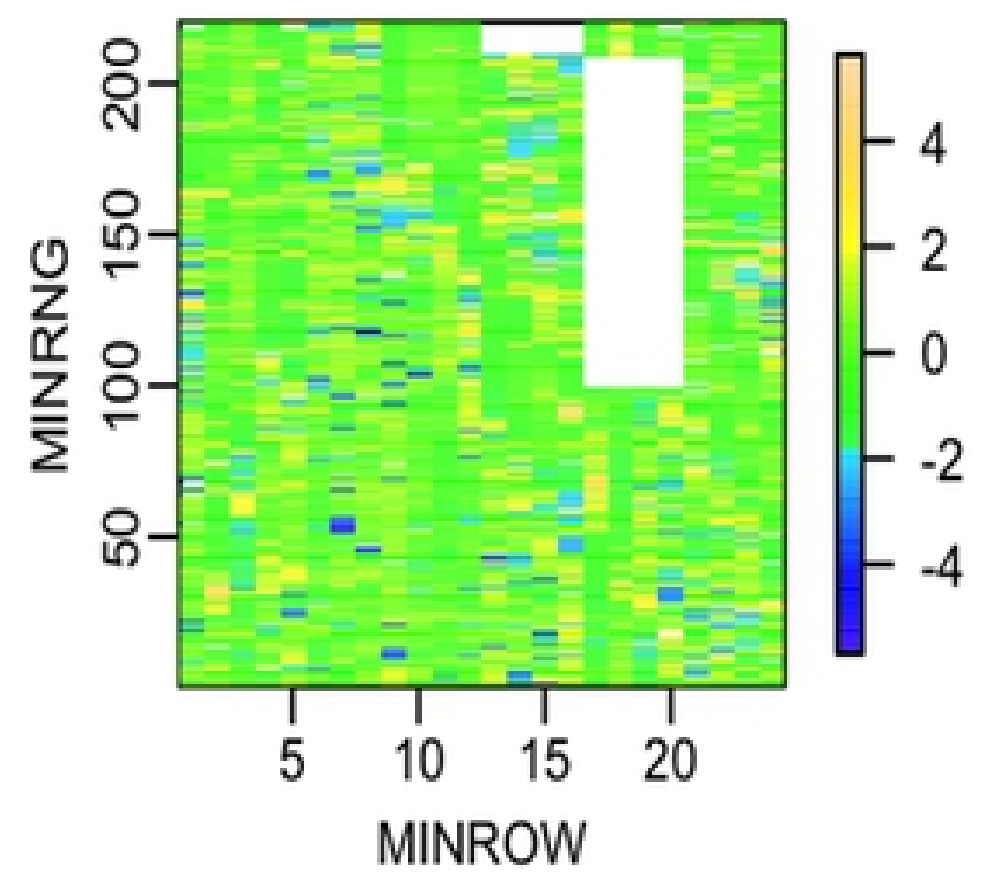

Histogram
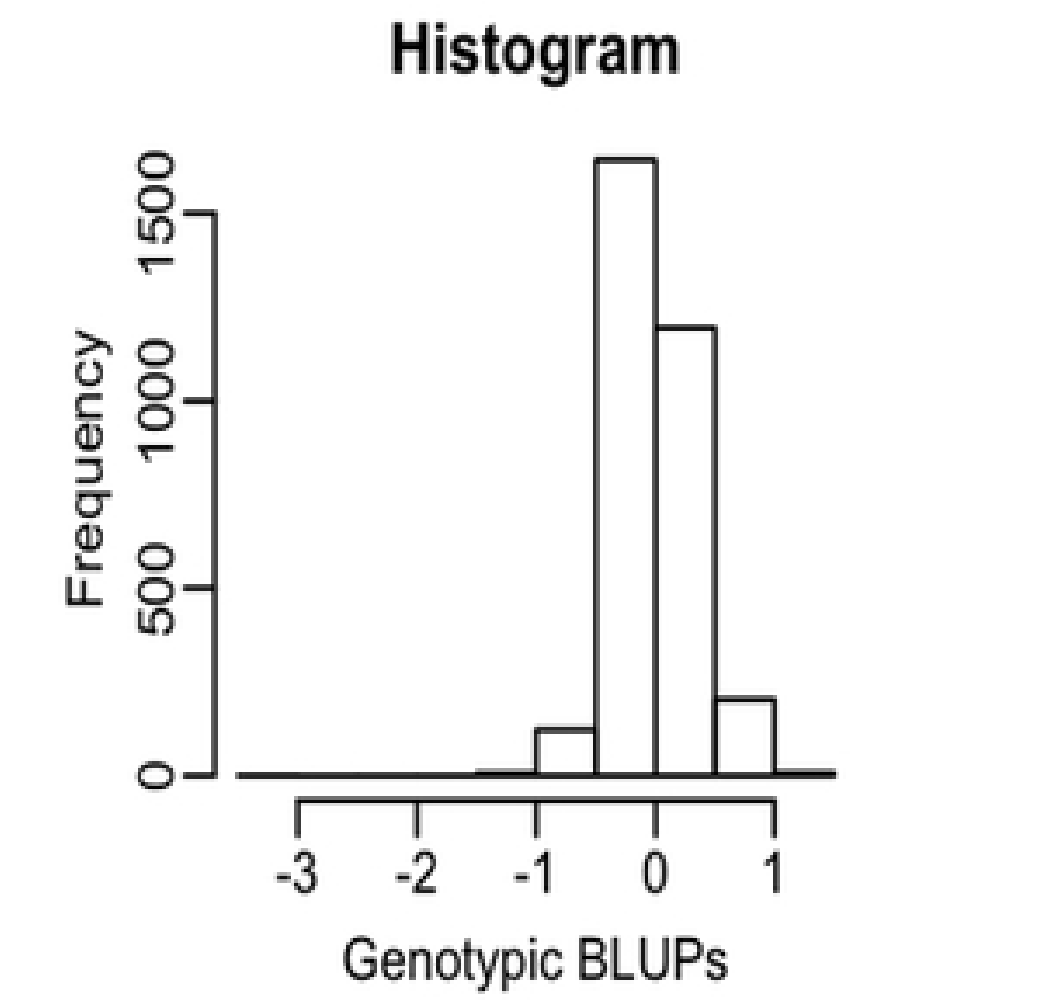

Figure
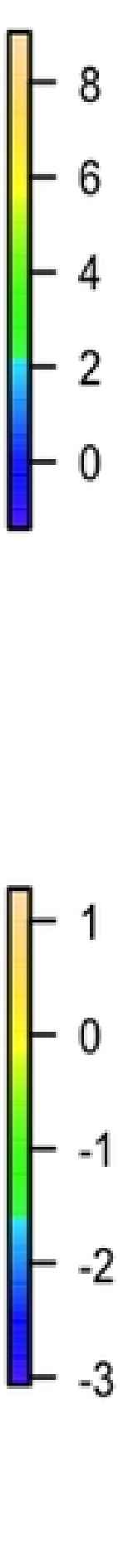

2 